This item was submitted to Loughborough's Research Repository by the author.

Items in Figshare are protected by copyright, with all rights reserved, unless otherwise indicated.

\title{
Optimal shape of an underwater moving bottom generating surface waves ruled by a forced Korteweg-de Vries equation
}

\section{PLEASE CITE THE PUBLISHED VERSION}

https://doi.org/10.1007/s10957-018-1400-8

\section{PUBLISHER}

(c) Springer Verlag

\section{VERSION}

AM (Accepted Manuscript)

\section{PUBLISHER STATEMENT}

This paper was accepted for publication in the journal Journal of Optimization Theory and Applications and the definitive published version is available at https://doi.org/10.1007/s10957-018-1400-8

\section{LICENCE}

CC BY-NC-ND 4.0

\section{REPOSITORY RECORD}

Dalphin, Jeremy, and Ricardo Lopes-Barros. 2019. "Optimal Shape of an Underwater Moving Bottom Generating Surface Waves Ruled by a Forced Korteweg-de Vries Equation”. figshare.

https://hdl.handle.net/2134/35138. 


\title{
Optimal Shape of an Underwater Moving Bottom \\ Generating Waves with a Forced Korteweg-de Vries Equation
}

\author{
Jeremy Dalphin • Ricardo Barros
}

Received: date / Accepted: date

\begin{abstract}
It is well known since $\mathrm{Wu} \& \mathrm{Wu}(1982)$ that a forcing disturbance moving steadily with a transcritical velocity in shallow water can generate, continuously and periodically, a succession of solitary waves propagating ahead of the disturbance in procession. One possible new application of this phenomenon could very well be surfing competitions, where in a controlled environment, such as a pool, waves can be generated with the use of a translating bottom. In this paper, we use the forced Korteweg-de Vries equation to investigate the shape of the moving body capable of generating the highest first upstream-progressing solitary wave. To do so, we study the following optimization problem: maximizing the total energy of the system over the set of non-negative square-integrable bottoms, with uniformly bounded norms and compact supports. We establish analytically the existence of a maximizer saturating the norm constraint, derive the gradient of the functional, and then implement numerically an optimization algorithm yielding the desired optimal shape.
\end{abstract}

Keywords Shape optimization · Existence theory · Optimal control · Surface wave generation · Numerical simulation · Forced Korteweg-de Vries equation · Finite-difference Methods

Mathematics Subject Classification (2000) primary 49K20 - secondary $35 \mathrm{Q} 53 \cdot 49 \mathrm{M} 29 \cdot 65 \mathrm{M} 06 \cdot 76 \mathrm{~B} 15 \cdot 49 \mathrm{~J} 45 \cdot 49 \mathrm{~J} 50$

Communicated by Grégoire Allaire

Jeremy Dalphin, Corresponding author

Centro de Modelamiento Matemático (CMM), Facultad de Ciencas Físicas y Matemáticas, UMR 2071 CNRS-Universidad de Chile, Beauchef 851, Santiago, Chile.

jdalphin@dim.uchile.cl

Ricardo Barros

Department of Mathematical Sciences, Loughborough University,

Loughborough LE11 3TU, United Kingdom.

r.barros@lboro.ac.uk 


\section{Introduction}

The generation of water-waves is a complex phenomenon and the artificial reproduction of such processes has many interesting applications for the engineering industry. In this paper, we consider a specific mechanism, initially discovered by $\mathrm{Wu} \& \mathrm{Wu}$ [1], which has recently been used to develop a patented wavemaker prototype [2]. The operating principle consists in translating a moving bottom underwater to produce waves upstream the forcing disturbance [3], which could have a new important application to surfing competitions, where in a controlled environment, such as a pool, waves can be generated at low operating costs (see [2]).

With this specific application in mind, the translating bottom is assumed to move steadily in shallow water with a transcritical velocity. We are interested in a reduced long-wave phenomenon and weakly dispersive as well as nonlinear effects will be considered, since experiments display the successive and continuous propagation of solitary waves ahead the moving disturbance. As shown by $\mathrm{Wu}$ in [3], under such conditions, the free surface can be effectively described by the forced Korteweg-de Vries (fKdV) equation. The realm of validity of this model has been extensively studied in the literature and it has been shown to be in good agreement, for a wide range of parameters, with laboratory experiments and numerical solutions of much more elaborate models (the generalized Boussinesq, fully non-linear Euler, and Navier-Stokes equations). We refer to [3-6] and references therein.

In this article, we investigate how the shape of an underwater moving bottom can affect the amplitude of the first upstream-progressing solitary wave. Under some practical constraints, we wish to find an efficient wave maker capable of generating the highest possible wave. This will be accomplished by considering the optimization problem (2), which consists on maximizing the total energy of the system.

Paramount to this study is the well-posedness of the fKdV equation with homogeneous initial data (see eq. (4)). The time global well-posedness in $H^{s}(\mathbb{R}, \mathbb{R})$, with $s \geqslant 0$, has been established by Bona \& Zhang [7]. The extension of this result to lower regularity forcing functions is not straightforward since, contrary to the case when $s \geqslant 0, L^{2}$-conservation laws are absent when $s<0$. Nonetheless, in the particular case when the flow is critical, the fKdV equation is endowed with a scaling property (Lemma B.1) that can be used to obtain a priori estimates as in the KdV case (see $[8,9]$ ). These rather elaborate techniques, combined with local well-posedness results known in the literature $[7,10]$, allowed Tsugawa [9] to prove the existence of a unique global solution to the initial-value problem (4) for low-regular bottoms.

We recall that there is a vast literature about the controllability and stabilization of the KdV equation (see e.g. [11] and references therein). However, the control problems related to the $\mathrm{KdV}$ equation are usually studied on a bounded interval, a periodic domain, or the half-line. To our knowledge, the control of the $\mathrm{KdV}$ equation by its second member on the whole real line has not been studied in the literature. Another novelty is that, although the 
non-linear problem (2) is stated in terms of an optimal control problem, it is studied here by adopting a shape optimization viewpoint.

A related problem was recently addressed by Nersisyan et al. [12]. In their work, opposed to the one presented here, the bottom velocity is not necessarily constant, thus both the piston trajectory and shape of the underwater wave maker are optimized, subject to practical constraints. The underlying mathematical model of their study is the generalized Benjamin-Bona-Mahony (BBM) equation, which similarly to the fKdV equation can be deduced from the generalized Boussinesq equations $[3,13]$. (1) becomes a distributed $L^{2}$ control function inside the infinite domain $\mathbb{R}$ of the $\mathrm{fKdV}$ equation (4). In that form, the optimization of the bottom shape is modelled by the optimal control problem (2). We refer to the references given in [12] for other applications of control theory to the BBM and $\mathrm{KdV}$ equations.

One of the reasons to retain the fKdV model in the present study is the mathematical challenges it presents, given that:

(i) theoretically, the existence of a maximizer to (2) for low-regular bottoms is not straightforward, as it is the case for smoother ones [12, Theorem 2];

(ii) numerically, the fKdV equation is known to be quite unstable, being its regularized version often preferred for computations [3].

These will be carefully investigated in this paper organized as follows. In $\S 2$, we introduce some necessary notation, formulate the optimization problem, and state our main result in Theorem 2.1, which establishes the existence of maximizer saturating the $L^{2}$-constraint. In $\S 3$ we present the mathematical model and summarize briefly the results in the literature. The optimization problem is then thoroughly discussed in $\S 4$. In $\S 5$, we provide an efficient numerical algorithm yielding an optimal shape. This consists of an Usawatype algorithm that converges iteratively to a critical point of (2), thanks to the evaluation of a shape gradient, which requires an adjoint formulation of the $\mathrm{fKdV}$ equation. The $\mathrm{fKdV}$ solver is also tested against other well-known numerical methods [16-18] regarding its efficiency and accuracy. Finally, the obtained numerical results are presented and discussed in $\S 6$, including the behaviour of the optimal shape with respect to its initial guess, the location and length of its support, and some open problems and perspectives.

\section{Problem Formulation}

In this study, the admissible moving bottoms will be required to belong to the set $\mathcal{B}$ defined as:

$$
\mathcal{B}:=\left\{b \in L^{2}(\mathbb{R}, \mathbb{R}): \operatorname{supp} b \subseteq[-K, K], b \geqslant 0 \text { and }\|b\|_{L^{2}(\mathbb{R}, \mathbb{R})} \leqslant M\right\},
$$

where $K, M$ are fixed positive constants, and $\|b\|_{L^{2}(\mathbb{R}, \mathbb{R})}:=\left(\int_{\mathbb{R}} b^{2}(x) \mathrm{d} x\right)^{1 / 2}$. We then propose an energy functional $F$ for which the following optimization problem is considered:

$$
\sup _{b \in \mathcal{B}} F(b) .
$$


The functional $F: b \in \mathcal{B} \mapsto F(b) \in \mathbb{R}$ is defined by

$$
F(b):=\left\|u_{b}\right\|_{L^{2}\left(0, T ; L^{2}(\mathbb{R}, \mathbb{R})\right)}^{2}=\int_{0}^{T} \int_{\mathbb{R}} u_{b}^{2}(x, t) \mathrm{d} x \mathrm{~d} t,
$$

where $T>0$ is fixed and $u_{b}(x, t)$ denotes the free-surface elevation ruled by a $\mathrm{fKdV}$ equation with zero initial data:

$$
\begin{cases}\frac{\partial u_{b}}{\partial t}+u_{b} \frac{\partial u_{b}}{\partial x}+\frac{\partial^{3} u_{b}}{\partial x^{3}}=-\frac{\mathrm{d} b}{\mathrm{~d} x} \in H^{-1}(\mathbb{R}, \mathbb{R}), & x \in \mathbb{R}, t \geqslant 0, \\ u_{b}(x, 0)=0, & x \in \mathbb{R} .\end{cases}
$$

As usual, the partial derivatives in (4) are understood in a distributional sense. For convenience, we assume the dimensionless form of all variables and functions. Moreover, the problem is set in the body frame of reference and, following common practice, $x$ and $t$ denote space and time coordinates, respectively.

We shall prove in Proposition 4.1 that (3) is a well-defined application from $L^{2}(\mathbb{R}, \mathbb{R})$ into $\mathbb{R}$. The choice of admissible bottoms (1) deserves some explanation, but, as it will be made clear, it turns out to be a rather natural one. Clearly, non-negative bottoms with compact support must be considered for manufacturing purposes. Also, the constraint imposed on the support guarantees the continuity of the functional (3) with respect to the $L^{2}$-weak topology (Proposition 4.3). Furthermore, from the physics viewpoint, to remain within the regime of validity of the mathematical model, the forcing function $b$ must be small enough. In that regard, an $L^{\infty}$-bound on the control function $b$ could have been imposed, rather than an $L^{2}$-one. However, numerical simulations suggest that an $L^{\infty}$-version of problem (2) would simply yield the step function saturating the constraint, which, from a realistic perspective, would be a very unsatisfactory wavemaker. We also show in Proposition B.4 that by discarding such an $L^{2}$-norm constraint, (2) becomes an ill-posed problem in the sense that the supremum is not achieved, even with smooth forcing functions.

Theoretically speaking, one could argue that it would have been more natural to consider the fKdV equation (4) in the $L^{2}$-sense, and thus choose an $H^{1}$-setting for (1). However, a corresponding numerical algorithm based on a steepest descent method would compute an irregular Lagrangian perturbation $\partial_{b} \mathcal{L}=\partial_{b} F-2 \lambda\left(b-b_{x x}\right) \in H^{-1}(\mathbb{R}, \mathbb{R})$. In comparison, the set of admissible shape (1) is stable with respect to such gradient perturbations, since, in this case, we will simply prove that $\partial_{b} \mathcal{L}=\partial_{b} F-2 \lambda b \in L^{2}(\mathbb{R}, \mathbb{R})$.

One of our main contributions is the proof of existence of an optimal bottom saturating the $L^{2}$-constraint, i.e. the following result holds:

Theorem 2.1 Let $T>0, M>0$, and $K>0$. We consider the set $\mathcal{B}$ and the functional $F: b \in \mathcal{B} \mapsto F(b)$ defined in (1) and (3), respectively. Then, the optimization problem (2) is well posed in the following sense:

$$
\exists b^{o p t} \in \mathcal{B}, \quad F\left(b^{o p t}\right)=\max _{b \in \mathcal{B}} F(b) .
$$

Moreover, any maximizer bopt of (2) satisfies $\left\|b^{o p t}\right\|_{L^{2}(\mathbb{R}, \mathbb{R})}=M$. 
This is a rather unexpected result since in our $H^{-1}$-setting, the lack of conservation laws for (4) could in principle prevent obtaining an explicit $C_{t}^{0}\left(H_{x}^{2}\right)$ control of the solution $u_{b}$ in terms of the $L^{2}$-norm of the bottom $b$. Nevertheless, we manage to obtain such a priori estimates (Proposition 4.2) by combining Tsugawa's results [9] with technical relations inspired from the conserved quantities available in the KdV case. Another main difficulty related to the lower-semi-continuity of the functional (3) is the lack of compactness of the embedding $H^{2}(\mathbb{R}, \mathbb{R}) \subset H^{1}(\mathbb{R}, \mathbb{R})$, so that standard arguments of AubinLions-Simon Lemma [14, Section 8 Corollary 4] cannot apply. We succeed in overcoming this difficulty by working directly with the non-linear equation (4) thanks to an integration-by-part technique available for Sobolev-Bochner spaces [15, Lemma 7.3], which is also used to prove the Fréchet differentiability of (3) (Proposition 4.5).

Another important contribution is the description in $\S 5$ of an efficient numerical algorithm providing an optimal shape, which could be useful for practical applications.

\section{Mathematical Model}

Consider a layer of water initially uniform in depth. Suppose we introduce at the bottom floor a disturbance moving steadily with speed $U$. Then, depending on the magnitude of $U$, different responses of this fluid-mechanical system can be obtained. One, particularly intriguing, arises when the flow is transcritical. As discovered by $\mathrm{Wu} \& \mathrm{Wu}[1]$, near resonance, a long-wave phenomenon takes place and solitary waves, propagating ahead of the disturbance, are periodically generated. Although originally discovered numerically based on the generalized Boussinesq equations, it is desirable to have a simpler model to carry out analytical and numerical investigations of the phenomenon. One such model, known as forced Korteweg-de Vries equation, was proposed by $\mathrm{Wu}$ [3] and it consists on a one-directional, weakly non-linear and weakly dispersive long wave model that is able to retain much of the physics of the problem. The model governs the free-surface elevation $\zeta(x, t)$ and reads in dimensional variables as follows:

$$
\frac{2}{c_{0}} \frac{\partial \zeta}{\partial t}-2\left(\frac{U}{c_{0}}-1\right) \frac{\partial \zeta}{\partial x}+\frac{3}{h_{0}} \zeta \frac{\partial \zeta}{\partial x}+\frac{h_{0}^{2}}{3} \frac{\partial^{3} \zeta}{\partial x^{3}}+\frac{\mathrm{d} b}{\mathrm{~d} x}=0
$$

For our purposes, $U$ is assumed positive and the equation holds for rightgoing waves. Here $t(\geqslant 0)$ is the time, $x=X-U t$ is the horizontal space variable expressed in the body frame of reference, $z=-h_{0}+b(x)$ indicates a topography $b(x)$ moving over the bottom floor at depth $h_{0}$, and $c_{0}$ is linear long wave speed defined as $c_{0}:=\sqrt{g h_{0}}$, with $g$ the gravitational acceleration.

As shown by [4-6], the model has a surprisingly wide range of validity, and a remarkable agreement with experiments and numerics for the fully non-linear Euler equations is achieved for $0.9<U / c_{0}<1.1$ and $\left\|b / h_{0}\right\|_{L^{\infty}(\mathbb{R}, \mathbb{R})}<0.15$. Moreover, if the translating bottom is sufficiently regular and the motion is 
assumed to start impulsively from rest, then it can be shown that the initial value problem for (5) is well-posed:

Lemma 3.1 Let $T, U, h_{0}$ be given fixed positive constants, and $b$ a sufficiently regular bottom, such that $b \in H^{\infty}(\mathbb{R}, \mathbb{R}):=\cap_{s \geqslant 0} H^{s}(\mathbb{R}, \mathbb{R})$. Then, there exists a unique solution $\zeta \in C^{\infty}\left(0, T ; H^{\infty}(\mathbb{R}, \mathbb{R})\right)$ of $(5)$ with zero initial data.

The proof consists in applying [7, Theorem 1.1] and using the fKdV equation (5) to gain, recursively, regularity in time. In $\S 4.1$, this well-posedness result will be extended for lower regular forcing functions.

Near resonance, the generic behaviour of the free-surface elevation predicted by the fKdV equation with zero initial data can be depicted as in Fig. 1. According to $\mathrm{Wu}[3]$, five different regions can be distinguished: some uniform states of depth $h_{0}$ far upstream and downstream on $\left.]-\infty, x_{0}\right] \sqcup\left[x_{2},+\infty[\right.$; a cnoidal-like ${ }^{1}$ wavetrain downstream on $\left[x_{0}, x_{1}\right]$; an almost uniform state of depth $h_{1}\left(<h_{0}\right)$ behind the disturbance on $\left[x_{1},-K\right]$; periodic succession of upstream advancing solitary waves on $\left[K, x_{2}\right]$.

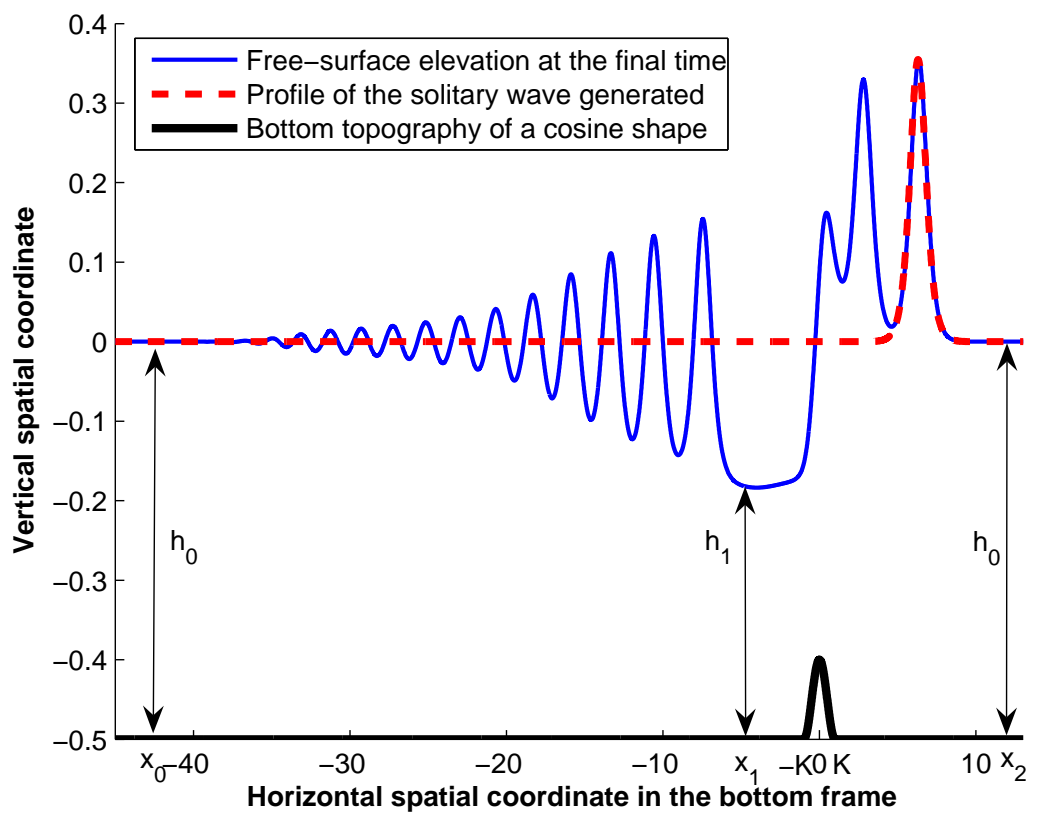

Fig. 1 Illustration of the generic behaviour of the solutions to the fKdV equation (5) near resonance. The translating body at the floor bottom has a cosine shape and moves at transcritical speed, i.e. $U / c_{0} \approx 1$. For comparison, the profile of a solitary-wave solution $\zeta_{\mathrm{KdV}}(x, t)$ (dashed line) in (7) is superposed to the first upstream-progressing wave.

\footnotetext{
1 Here, the term cnoidal simply refers to the profile of the periodic travelling-wave solutions to the $\mathrm{KdV}$ equation (see [19]).
} 
For simplicity, here and hereafter, we shall limit ourselves to the critical case of $U=c_{0}$. In this situation, the $\mathrm{fKdV}$ equation reduces to

$$
\frac{2}{c_{0}} \frac{\partial \zeta}{\partial t}+\frac{3}{h_{0}} \zeta \frac{\partial \zeta}{\partial x}+\frac{h_{0}^{2}}{3} \frac{\partial^{3} \zeta}{\partial x^{3}}+\frac{\mathrm{d} b}{\mathrm{~d} x}=0
$$

and can be cast, by a simple change of variables, into the canonical form given in (4), better suited to the theoretical study of the optimization problem (2):

Lemma 3.2 Set $\mathfrak{x}:=3^{3 / 5} x / h_{0}$ and $\mathfrak{t}:=3^{4 / 5} c_{0} t /\left(2 h_{0}\right)$. Consider the functions $u(\mathfrak{x}, \mathfrak{t}):=3^{4 / 5} \zeta(x, t) / h_{0}$ and $\mathfrak{b}(\mathfrak{x}):=3^{3 / 5} b(x) / h_{0}$. Then $u(\mathfrak{x}, \mathfrak{t})$ is a solution of (4) with forcing term $\mathfrak{b}(\mathfrak{x})$ if and only if $\zeta(x, t)$ solves $(6)$ with zero initial data.

Remark that the classical KdV equation can be recovered from (6) when the forcing disturbance vanishes, i.e. $b=0$, in which case we have the well-known family of solitary-wave solutions

$$
\zeta_{\mathrm{KdV}}(x, t)=a \operatorname{sech}^{2}\left[\sqrt{\frac{3 a}{4 h_{0}^{3}}}\left(x-x_{0}-\frac{a c_{0}}{2 h_{0}} t\right)\right] .
$$

Figure 1 illustrates how well the "runaway" solitons of the fKdV equation can be captured by the classical soliton profile (7) for the $\mathrm{KdV}$ equation. If the motion starts impulsively from rest, we observe that it takes a certain time $T_{g}$ until the first upstream-progressing solitary wave is fully formed and breaks away from the disturbance. As time evolves, multiple (almost identical) copies of the leading wave will be produced in a periodic way. If $a$ is the amplitude of such upstream-progressing solitary waves, then the period of generation $T_{s}$ can be estimated accordingly to $\mathrm{Wu}[3]$ by

$$
T_{s}=\frac{64 h_{0}}{c_{0}}\left(\frac{h_{0}}{3 a}\right)^{\frac{3}{2}} .
$$

The formula reveals a somewhat counterintuitive feature of the system: the higher the amplitude of the upstream waves, the shorter the period of generation $T_{s}$. The times $T_{g}$ and $T_{s}$ are somewhat related and numerical evidence seem to suggest that $T_{g}$ is less (greater) than $T_{s}$ for positive (negative) forcing functions (see e.g. [3]).

The presence of a non-constant forcing term in (6) has the effect of destroying the invariance with respect to spatial translations, and so the excess energy integral $\frac{1}{2} \int_{\mathbb{R}} \zeta^{2} \mathrm{~d} x$ is not a constant of motion. Indeed, by multiplying (6) by $\zeta$ and integrating the resulting equation over the real line, one obtains

$$
\frac{\mathrm{d}}{\mathrm{d} t} \int_{\mathbb{R}} \zeta^{2} \mathrm{~d} x=c_{0} D_{w},
$$

where $D_{w}$ is given by $D_{w}(t):=\int_{\mathbb{R}} b(x) \partial_{x} \zeta(x, t) \mathrm{d} x$ and can be physically interpreted as the drag, or the resistance due to unsteady wave making. As a consequence, we obtain

$$
\forall t>0, \quad\|\zeta(\bullet, t)\|_{L^{2}(\mathbb{R}, \mathbb{R})}^{2}=c_{0} \int_{0}^{t} D_{w}(s) \mathrm{d} s .
$$


Furthermore, numerics strongly suggest that the function $t \mapsto\|\zeta(\bullet, t)\|_{L^{2}(\mathbb{R}, \mathbb{R})}^{2}$ can be reasonably approximated by $t \mapsto c_{0} \bar{D}_{w} t$, with $\bar{D}_{w}$ the average value of $D_{w}$ over the wave period $T_{s}$. By mass and energy considerations, such value can also be estimated as $\bar{D}_{w}=a^{3} /\left(4 h_{0}\right)$ (see [3]). Hence, we can say that qualitatively

$$
\int_{0}^{T} \int_{\mathbb{R}} \zeta^{2}(x, t) \mathrm{d} x \mathrm{~d} t \quad \text { and } \quad c_{0} \bar{D}_{w} \frac{T^{2}}{2}=\frac{c_{0} T^{2} a^{3}}{8 h_{0}}
$$

have similar behaviours, provided the elapsed time $T$ exceeds the time $T_{g}$ necessary to allow the generation of the first upstream solitary wave.

\section{Optimization Problem}

For the purposes of our work, the flow is assumed to be critical and properties of the corresponding fKdV solutions are exploited to formulate the optimization problem. For convenience, the problem is stated in terms of nondimensional variables as in $\S 2$. In particular, the parameters in (1) must be chosen appropriately to remain within the range of validity of the fKdV model: we set $K:=K_{\operatorname{dim}} / h_{0}=O(1)$ and $M=M_{\operatorname{dim}} / h_{0}=O\left(10^{-1}\right)$. Moreover, for obvious manufacturing reasons, only non-negative forcing functions with compact support will be admitted.

An efficient wave maker is one capable of generating a wave of high amplitude. Since the dynamics is well described by the fKdV model, we can rely on the description given in Fig. 1 to assert that from the moment the wave maker starts moving steadily at critical speed, an upstream-progressing solitary wave will be generated after a certain time $T_{g}$. This wave will suddenly unlatch from the moving bottom and will propagate throughout large distances without altering its form. It depends on one single parameter (its amplitude $a$ ) and moves with an excess speed $c=a c_{0} / 2 h_{0}$ relative to the bottom $(c f$. . (7) in dimensional variables). From a practical point of view, once the leading wave is fully formed, the wave maker could then be stopped, without that same wave being affected. In the surfing context, this elevation wave would be the wave of interest, and the one that we wish to maximize. In other words, we would like to solve

$$
\sup _{\substack{b \in C_{c}^{\infty}(\mathbb{R},[0,+\infty[) \\ \operatorname{supp} b \subseteq[-K, K]}} a(b) .
$$

Moreover, we can use (10) to argue that, at least qualitatively, maximizing the functional $b \mapsto a(b)$ should yield similar results to those obtained by maximizing the functional $b \mapsto F(b)$ given in (3). Alternatively, one may address the problem

$$
\sup _{\substack{b \in C_{c}^{\infty}(\mathbb{R},[0,+\infty[) \\ \operatorname{supp} b \subseteq[-K, K]}} \int_{0}^{T} \int_{\mathbb{R}} u_{b}^{2}(x, t) \mathrm{d} x \mathrm{~d} t
$$


where $u_{b}$ is the solution corresponding to the forcing function $b$ in (4), and where the choice of a final time $T>0$ is discussed below. The formulation (11), which is a smooth unconstrained version of (2), also allows us to carry out the numerical procedure developed in $\S 5.1$, since the employed gradient method relies on the directional derivative of the functional (3). Furthermore, the $L^{2}$-setting is considered for the set (1) of admissible bottoms, ensuring the numerical stability of the optimization algorithm. In particular, an $L^{2}$-norm constraint on the forcing functions is imposed, otherwise the supremum is not achieved, cf. Appendix $B$.

We now comment the choice of a final time $T$. One could be tempted to examine the asymptotic behaviour of (2) as $T \rightarrow+\infty$. Indeed, for problems involving conservation laws and propagation phenomena, the introduction of a finite time $T$ (and a backward adjoint system) can potentially create undesirable artificial and numerical phenomena. Consequently, it would be more convenient to replace a time-dependant optimal control problem by the timeindependent one associated with its asymptotic behaviour. The issue is important and still open, apart from some recent work of Trélat \& Zuazua [20], where in some cases, they estimate the error made on such approximation. However, in the considered setting, we have some good reasons not do so.

Heuristically, from (10), regardless the bottom considered, the integral over time of the energy should go to infinity as $T \rightarrow+\infty$. In addition, the dynamics of our system is completely determined in advance. As described in $\S 3$, once the time $T_{g}$ is reached and a first upstream-progressing solitary wave is produced, copies of such wave are generated every interval of time of length $T_{s}$, given by (8). All of these propagate ahead of the translating bottom and remain permanent in form, regardless whether or not the bottom keeps its procession. This periodic pattern in time of the system motivates the picking of a finite time $T$. Lastly, in real applications, the phenomenon takes place in a finite pool, so that the final time $T$ must also be finite to ensure that some waves, potentially reflected from the pool ends, do not affect our system. We address the reader to $\S 5.2$ for an algorithmic description of how $T$ is chosen in practice.

To prove Theorem 2.1, a number of steps will be introduced in this section. Following closely Tsugawa [9], we start by establishing in $\S 4.1$ the time global well-posedness of (4) and proving that the functional $F$ proposed in (3) is welldefined. We then proceed by providing in $\S 4.2$ some explicit a priori estimates that allow us to prove the existence of an optimal bottom in $\S 4.3$. Finally, in $\S 4.4$ the Fréchet differentiability of (3) is established giving us access to the gradient of (3) via the adjoint formulation of (4). All the proofs and technical details are included in Appendix A and B.1 for a better readability.

\subsection{Global Well-Posedness of the fKdV Equation}

We start by recalling the following result of Tsugawa [9, Theorem 1.2]: the initial value problem for the equation $\partial_{t} u+u \partial_{x} u+\partial_{x x x} u=f(x) \in H^{\sigma}(\mathbb{R}, \mathbb{R})$ 
with $\sigma \geqslant-\frac{3}{2}$ and $\left.\left.u(\bullet, 0) \in H^{s}(\mathbb{R}, \mathbb{R}), s \in\right]-\frac{3}{4}, \sigma+3\right]$ is globally well-posed in time. We also mention that the proof of this result not trivial and uses a rather sophisticated tool: the $I$-method developed by T. Tao et al. [8].

Although there is no conservation law for the (f) KdV equation in $H^{s}(\mathbb{R})$ for any $s<0$, we have managed to perform on (4) a kind of integration-bypart technique for Sobolev-Bochner space [15, Lemma 7.3] still available in our $H^{-1}$-setting. As a consequence, we are able to recover an estimate, merely giving the sequential continuity of the map $b \in L^{2} \mapsto u_{b} \in C_{t}^{0}\left(L_{x}^{2}\right)$.

Proposition 4.1 Let $T>0$ and $b \in L^{2}(\mathbb{R}, \mathbb{R})$. Then, the initial-value problem (4) is well posed i.e. it has a unique global solution $u_{b} \in C^{0}\left(0, T ; H^{2}(\mathbb{R}, \mathbb{R})\right)$. Moreover, given any other pair $\left(\mathfrak{b}, u_{\mathfrak{b}}\right)$ formed by a forcing term $\mathfrak{b} \in L^{2}(\mathbb{R}, \mathbb{R})$ and its corresponding solution $u_{\mathfrak{b}} \in C^{0}\left(0, T ; H^{2}(\mathbb{R}, \mathbb{R})\right)$, we have:

$$
\sup _{t \in[0, T]}\left\|u_{\mathfrak{b}}(\bullet, t)-u_{b}(\bullet, t)\right\|_{L^{2}(\mathbb{R}, \mathbb{R})}^{2} \leqslant 4 C T e^{C T}\|\mathfrak{b}-b\|_{L^{2}(\mathbb{R}, \mathbb{R})},
$$

where $C:=\max \left(\left\|u_{b}\right\|_{C^{0}\left(0, T ; H^{2}(\mathbb{R}, \mathbb{R})\right)},\left\|u_{\mathfrak{b}}\right\|_{C^{0}\left(0, T ; H^{2}(\mathbb{R}, \mathbb{R})\right)}\right)$. In particular, the functional $F(b)$ in $(3)$ is a well-defined application from $L^{2}(\mathbb{R}, \mathbb{R})$ into $\mathbb{R}$.

\subsection{Explicit A Priori Estimates}

Again, the absence of conservation laws for (4) gives few hope for an explicit $C_{t}^{0}\left(H_{x}^{2}\right)$-control of the solution $u_{b}$ in terms of the $L^{2}$-norm of the bottom $b$. Nevertheless, such an estimate is fundamental for the theoretical study of (2). Fortunately, Tsugawa was able to prove in [9] that a global $C_{t}^{0}\left(L_{x}^{2}\right)$-estimate is achieved, with an explicit expression in terms of final time and second-member.

One of our important contributions here has been to improve his estimate by discovering some relations for the fKdV case with smooth data, namely (20)-(21), inspired from the conserved quantities of the KdV equation. However, (20)-(21) can only hold under the assumption that $b$ and $u_{b}$ have enough regularity, so that we can differentiate under the integral sign and commute the order of the mixed partial derivatives.

Therefore, we have to proceed in two steps. In Proposition A.1, we first obtain an explicit global $C_{t}^{0}\left(H_{x}^{2}\right)$-control of $u_{b}$ in terms of the $L^{2}$-norm of $b$ but we have to assume that $b$ and $u_{b}$ are very smooth. Then, thanks to the the quantitative estimate of Proposition 4.1 and an approximation argument, we are able to extend the result as follows.

Proposition 4.2 Let $T>0$ and $b \in L^{2}(\mathbb{R}, \mathbb{R})$. Then, the unique solution $u_{b} \in C^{0}\left(0, T ; H^{2}(\mathbb{R}, \mathbb{R})\right)$ given in Proposition 4.1 satisfies the inequality:

$$
\begin{aligned}
\left\|u_{b}\right\|_{C^{0}\left(0, T ; H^{2}(\mathbb{R}, \mathbb{R})\right)} \leqslant P_{0}\left(T,\|b\|_{L^{2}(\mathbb{R}, \mathbb{R})}\right) & +P_{1}\left(T,\|b\|_{L^{2}(\mathbb{R}, \mathbb{R})}\right) \\
& +e^{T\left(1+\frac{1}{3}\|b\|_{L^{2}(\mathbb{R}, \mathbb{R})}^{2}\right)} P_{2}\left(T,\|b\|_{L^{2}(\mathbb{R}, \mathbb{R})}\right),
\end{aligned}
$$

where $P_{0}, P_{1}$, and $P_{2}$ are the polynomials introduced in Proposition A.1. 


\subsection{Existence of an Optimal Bottom}

To prove the existence of an optimal bottom, as in Theorem 2.1, a suitable topology must be introduced on the set of admissible bottoms ensuring:

- the compactness of any maximizing sequence of the supremum in (2);

- the closedness of the set of admissible bottoms (1);

- the (upper-semi)continuity of the energy functional (3).

From Proposition 4.1, the functional (3) is a well-defined map from $L^{2}(\mathbb{R}, \mathbb{R})$ into $\mathbb{R}$, thus adopting the $L^{2}$-weak topology would seem rather natural. In addition, the compactness of $\mathcal{B}$ follows from the fact that positivity, uniform upper bound and support are preserved by the $L^{2}$-weak convergence.

Lemma 4.1 Let $K>0$ and $M>0$. Then, the set of admissible bottoms (1) is sequentially compact for the weak topology of $L^{2}(\mathbb{R}, \mathbb{R})$.

However, the continuity of the functional (3) is not straightforward. Indeed, we are now dealing with the weak convergence of bottoms and the results of $\S 4.2$ such as the quantitative estimate of Proposition 4.1 are useless since they involve the strong topology of $L^{2}(\mathbb{R}, \mathbb{R})$.

A standard technique to recover the some continuity usually consists in exploiting the a priori estimates thanks to a compact embedding, usually given by the Aubin-Lions-Simon Lemma [14, Section 8 Corollary 4]. However, it does not apply to our case because the embedding $H^{2}(\mathbb{R}, \mathbb{R}) \subset H^{1}(\mathbb{R}, \mathbb{R})$ is not compact. Nevertheless, we have managed to overcome this difficulty by using the fact the supports of the admissible bottoms (1) are all contained in a fixed compact set.

Proposition 4.3 Let $T>0, K>0$ and $b \in L^{2}(\mathbb{R}, \mathbb{R})$ with support included in $[-K, K]$. Consider any sequence $\left(b_{n}\right)_{n \in \mathbb{N}}$ of square-integrable maps with supports all included in $[-K, K]$ converging weakly to $b$ in $L^{2}(\mathbb{R}, \mathbb{R})$. Then, the sequence $\left(u_{b_{n}}\right)_{n \in \mathbb{N}}$ converges strongly to $u_{b}$ in $C^{0}\left(0, T ; L^{2}(\mathbb{R}, \mathbb{R})\right)$, where $u_{b}$ and $u_{b_{n}}$ are the unique maps of $C^{0}\left(0, T ; H^{2}(\mathbb{R}, \mathbb{R})\right)$ associated to $b$ and $b_{n}$, respectively, as in Proposition 4.1 for any $n \in \mathbb{N}$.

\subsection{Fréchet Differentiability of the Functional}

In this section, we derive the Fréchet differentiability of $F$ with respect to $L^{2}$-perturbations. If the formal computation is quite straightforward, its proof requires careful arguments, such as the non-trivial continuity of the map $b \in$ $L^{2} \mapsto u_{b} \in C_{t}^{0}\left(H_{x}^{1}\right)$, studied in Appendix B.1. Hence, we obtain an explicit expression for the gradient of $F$ by introducing the adjoint formulation of (4).

Proposition 4.4 Let $T>0, b \in L^{2}(\mathbb{R}, \mathbb{R})$, and consider the unique solution $u_{b} \in C^{0}\left(0, T ; H^{2}(\mathbb{R}, \mathbb{R})\right)$ of Proposition 4.1 satisfying (4). Then, the following 
final-value problem (understood as a distributional equality) is well-posed:

$$
\begin{cases}\frac{\partial v}{\partial t}+u_{b} \frac{\partial v}{\partial x}+\frac{\partial^{3} v}{\partial x^{3}}+2 u_{b}=0 \in H^{-1}(\mathbb{R}, \mathbb{R}) & x \in \mathbb{R}, t \in[0, T] \\ v(x, T)=0 & x \in \mathbb{R},\end{cases}
$$

in the sense that it has a unique global solution $v_{b} \in C^{0}\left(0, T ; H^{2}(\mathbb{R}, \mathbb{R})\right)$.

Proposition 4.5 Let $T>0$ and $F: L^{2}(\mathbb{R}, \mathbb{R}) \rightarrow \mathbb{R}$ be well-defined by (3) (cf. Proposition 4.1). Then, for any $b \in L^{2}(\mathbb{R}, \mathbb{R})$ and any $h \in L^{2}(\mathbb{R}, \mathbb{R})$, the following expansion holds:

$$
F(b+h)=F(b)+\int_{\mathbb{R}} h(x)\left(\int_{0}^{T} \frac{\partial v_{b}}{\partial x}(x, t) \mathrm{d} t\right) \mathrm{d} x+o\left(\|h\|_{L^{2}(\mathbb{R}, \mathbb{R})}\right),
$$

where $v_{b} \in C^{0}\left(0, T ; H^{2}(\mathbb{R}, \mathbb{R})\right)$ is the unique global solution of $(12)$ introduced in Proposition 4.4. In particular, the map $F_{b}: h \in L^{2}(\mathbb{R}, \mathbb{R}) \mapsto F(b+h) \in \mathbb{R}$ is Fréchet differentiable at the origin for any bottom $b \in L^{2}(\mathbb{R}, \mathbb{R})$ and the gradient of $F$ at $b$ is given by

$$
\partial_{b} F: x \in \mathbb{R} \longmapsto \partial_{b} F(x):=\int_{0}^{T} \frac{\partial v_{b}}{\partial x}(x, t) \mathrm{d} t .
$$

Henceworth, the map $b \in L^{2}(\mathbb{R}, \mathbb{R}) \mapsto \partial_{b} F \in L^{2}(\mathbb{R}, \mathbb{R})$ is referred to as the shape gradient of $F$.

\section{Numerical Approach to the Problem}

In this section, we present the numerical procedure used to solve problem (2). In particular, we aim to develop a numerical scheme allowing a fast and precise resolution of the fKdV equation on account of being incorporated in the loop of the optimization algorithm. Here and hereafter, the dimensional form (6) for the fKdV equation will be used. All simulations in this work were performed on a standard laptop with Matlab and with fixed values $h_{0}=1 \mathrm{~m}$ and $g=9.81 \mathrm{~m} \cdot \mathrm{s}^{-2}$.

\subsection{Numerical Scheme for the fKdV Equation}

A naive discretization of equation (6) using finite differences is expected to perform rather poorly. Contributing to this is the fact that, most likely, some physical properties are not conserved, the third-order derivative introduces numerical dispersion, and the forcing term breaks some possible symmetries. For the KdV equation, which is just a particular case of (6), some well-known efficient methods can be found in the literature (see e.g. [16-18]). All these three methods are, however, subject to a drastic stability condition of the 
form $\Delta t=O\left(\Delta x^{3}\right)$. To overcome this inconvenience, Furihata proposes in [21] an implicit finite-difference scheme that is unconditionally stable. This numerical scheme is known to be very stable because it also preserves the physical properties of the equation, namely the mass and the Hamiltonian.

Such method can be easily adapted to discretize (6), as follows. Noticing that (6) can be cast into the form $\left(2 / c_{0}\right) \partial_{t} u=\partial_{x}\left(\delta_{u} G\right)$, where we have set $\delta_{u} G:=-\left(3 / 2 h_{0}\right) u^{2}-\left(h_{0}^{2} / 3\right) \partial_{x x} u-b$, we have

$$
G\left(\frac{\partial u}{\partial x}, u\right)=-\frac{1}{2 h_{0}} u^{3}+\frac{h_{0}^{2}}{6}\left(\frac{\partial u}{\partial x}\right)^{2}-b u .
$$

First, considering a small space step $\Delta x>0$ and a small time step $\Delta t>0$, the domain is uniformly discretized: $\mathbb{R} \times[0, T] \approx\left(x_{i}, t_{n}\right)_{(i, n) \in \mathbb{Z} \times \llbracket 0, N \rrbracket}$ with $x_{i}=i \Delta x, t_{n}=n \Delta t$ and $N \Delta t=T$. In the numerical scheme, $U_{i}^{n}$ approximates $u_{i}^{n}:=u\left(x_{i}, t_{n}\right)$. We then introduce the discrete operators $\delta_{i}^{+}(\bullet)=\left[(\bullet)_{i+1}-\right.$ $\left.(\bullet)_{i}\right] / \Delta x$ and $\delta_{i}^{-}(\bullet)=\left[(\bullet)_{i}-(\bullet)_{i-1}\right] / \Delta x$. According to the general procedure described by Furihata [21, Section 5.1], and adopting the same notation, we thus have $\left.G_{d}(U)_{i}:=-\left(1 / 2 h_{0}\right) U_{i}^{3}+\left(h_{0}^{2} / 12\right)\left[\delta_{i}^{+} U_{i}\right)^{2}+\left(\delta_{i}^{-} U_{i}\right)^{2}\right]-B_{i} U_{i}$, and so:

$$
\left\{\begin{array}{l}
\frac{\delta G_{d}}{\delta(U, V)_{i}}:=-\frac{U_{i}^{2}+U_{i} V_{i}+V_{i}^{2}}{2 h_{0}}-\frac{h_{0}^{2}}{6} \delta_{i}^{+} \delta_{i}^{-}\left(U_{i}+V_{i}\right)-B_{i} \\
\frac{2}{c_{0}} \frac{U_{i}^{n+1}-U_{i}^{n}}{\Delta t}=\frac{1}{2 \Delta x}\left(\frac{\delta G_{d}}{\delta\left(U^{n+1}, U^{n}\right)_{i+1}}-\frac{\delta G_{d}}{\delta\left(U^{n+1}, U^{n}\right)_{i-1}}\right) .
\end{array}\right.
$$

The remarkable feature of the above discretization is that the solutions obtained $\left(U_{i}^{n}\right)_{(i, n) \in \mathbb{Z} \times \llbracket 0, N \rrbracket}$ for this set of non-linear equations satisfy (see [21]):

$$
\forall n \in \llbracket 0, N \rrbracket, \quad \sum_{i \in \mathbb{Z}} G_{d}\left(U^{n}\right)_{i} \Delta x=0 \quad \text { and } \quad \sum_{i \in \mathbb{Z}} U_{n}^{i} \Delta x=0 .
$$

In other words, the scheme preserves, at a discrete level, the mass and the Hamiltonian structure of equation (6).

With the view of incorporating a fast numerical scheme into the loop of the optimization algorithm, we propose to simplify the numerical method by linearizing it thanks to the approximation $\left(u_{i}^{n+1}\right)^{2}+\left(u_{i}^{n}\right)^{2}=2 u_{i}^{n+1} u_{i}^{n}+O\left(\Delta t^{2}\right)$, valid for any $i \in \mathbb{Z}$ and $u\left(x_{i}, \bullet\right) \in C^{2}(\mathbb{R}, \mathbb{R})$. Our discretization of (6) amounts to solving the following linear system:

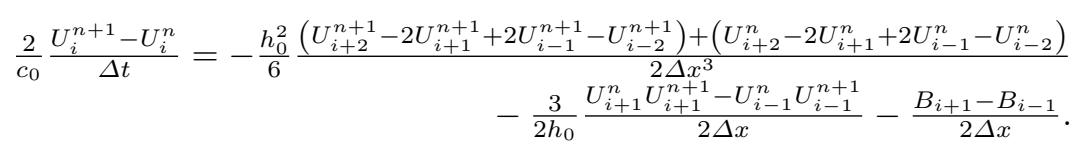

This will be the numerical scheme retained in this work, given that it offers a good compromise between a fast and accurate resolution and has some good numerical properties, $c f$. Appendix B.2. In particular, it is consistent and unconditionally stable, and preserves mass. 
To test the performance of our numerical scheme, we set $b=0$, in which case the $\mathrm{KdV}$ equation is recovered, and compare it against the efficient methods given in [16-18]. As we know, in the particular case when the forcing term is absent, we have a family of solitary-wave solutions $\zeta_{\mathrm{KdV}}$ defined by $(7)$. This traveling-wave solution corresponds to the solution of the KdV equation with initial condition $\zeta_{\mathrm{KdV}}(\bullet, 0)$, i.e. the initial profile remains unaltered as it propagates, which can be used as a benchmark test for the different schemes. The equation is solved numerically on $[-L, L] \times[0, T]$ with initial condition $\zeta_{\mathrm{KdV}}(\bullet, 0)$, by imposing periodic boundary conditions. The numerical errors obtained with respect to the exact solution and computational time required by each one of the methods are depicted in Fig. 2 .

As it can be seen from the figure, as long as the stability condition $\Delta t=$ $O\left(\Delta x^{3}\right)$ is required, although slower, our scheme is as precise as its competitors. However, thanks to Proposition B.3, such constraint can be relaxed, say by considering $\Delta t=O(\Delta x)$, allowing to drastically reduce the computational time while keeping the same order of precision, as shown in the last entry of the chart legend in Fig. 2.

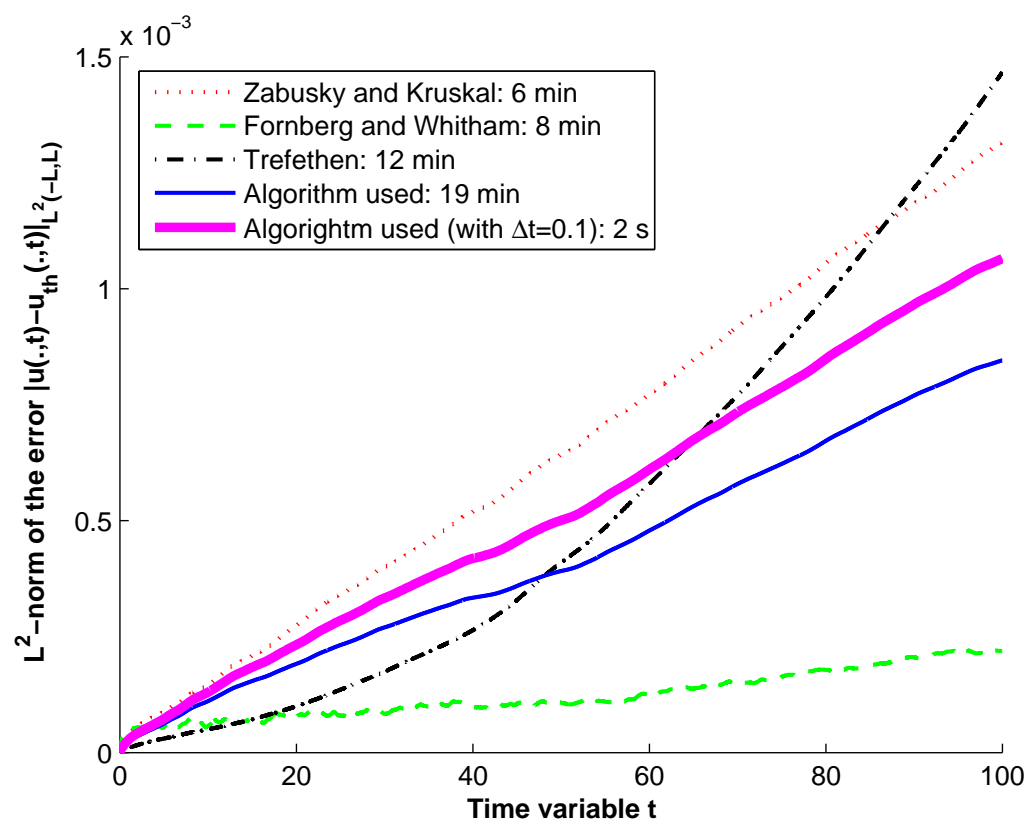

Fig. 2 Performance comparison between different numerical schemes for the KdV equation. The problem is solved on $[-L, L] \times[0, T]$ with periodic boundary conditions, and the numerical solutions are compared against the analytical solitary-wave solution. The computational time required by each one of the methods is specified in the chart legend. Parameters are set as: $a=0.2, x_{0}=0, \Delta x=0.1, L=15, \Delta t=0.00025$, and $T=100$. The last line of the chart legend has been obtained by changing the value of $\Delta t(=0.1)$ while keeping the same values of the other parameters. 


\subsection{Numerical Boundary Conditions}

In contrast to what was seen in $\S 5.1$, in the absence of a forcing term, we will solve equation (6) on a finite domain, say from $-L$ to $L$, without imposing periodic boundary conditions. Since the flow is assumed to be critical, a positive constant $L$ is chosen large enough to contain all the five distinct regions depicted in Fig. 1. We may then introduce $I \in \mathbb{N}$ such that $I \Delta x=L$ and we have $U_{i}^{n+1}=U_{i}^{n}=0$ for any $i \notin \llbracket-I, I \rrbracket$. In particular, the discretization (15) becomes a linear system of $2 I+1$ equations, which reads $A^{n} U^{n+1}=B^{n}$ in matrix form. Instead of increasing the execution time with the choice of a large $L$ that would depend on $T$, a smooth filter $f$ is applied on the approximated map at each time step. The introduction of a filter has the aim of suppressing the waves at the left-end of the domain $[-L,-L+\Delta L]$, with $\Delta L \in] 0, L[$ being the interval size on which the filter acts, while preserving the solution behavior outside this region. More precisely:

$$
\left\{\begin{array}{l}
U_{i}^{n+1}=f\left(x_{i}\right)\left[\left(A^{n}\right)^{-1} B^{n}\right]_{i} \\
f(x)=\frac{1}{2}\left[1+\cos \left(\pi \frac{\Delta L-(L+x)}{\Delta L}\right)\right] \mathbf{1}_{[-L,-L+\Delta L]}(x)+\mathbf{1}_{]-L+\Delta L, L]}(x),
\end{array}\right.
$$

where $\mathbf{1}_{[-L,-L+\Delta L]}$ (respectively $\mathbf{1}_{-L+\Delta L, L]}$ ) is valued one on $[-L,-L+\Delta L]$ (resp. $]-L+\Delta L, L]$ ), otherwise zero. This procedure ensures a smooth decreasing of $U^{n+1}$ to zero on $[-L,-L+\Delta L]$ and it does not affect the approximation of $u\left(\bullet, t_{n+1}\right)$ on $[-L+\Delta L, L]$ as shown in Fig. 3. More importantly, it allows a small numerical domain, which greatly reduces the computational time of our simulations. Finally, concerning the right-end of the domain, knowing that waves entering this region are solitary waves, we can use their explicit expression (7) and their generation period (8) in order to tune the final time $T$ and prevent them from reaching the boundary.

To choose the final time $T$, we proceed as follows. Consider an educated initial guess $b^{0}$ and a sequence of bottoms $b^{k}$ converging to an optimal one $b^{\text {opt }}$ thanks to an optimization procedure ensuring that the amplitudes of the corresponding "runaway" waves are not decreasing. In particular, we have $a\left(b^{0}\right) \leqslant a\left(b^{k}\right)$ and by $(8)$, we get $T_{s}\left(b^{0}\right) \geqslant T_{s}\left(b^{k}\right)$. Recalling that $T_{s}>T_{g}$, we deduce that $T_{s}\left(b^{0}\right)>T_{g}\left(b^{k}\right)$ for any $k \in \mathbb{N}$, and similarly $T_{s}\left(b^{0}\right)>T_{g}\left(b^{\text {opt }}\right)$. Note that $T_{s}\left(b^{0}\right)$ is explicit from (8) and a measure of $a\left(b^{0}\right)$ given by any simulation. Hence, any choice of $T$ greater than $T_{s}\left(b_{0}\right)$ is appropriate because it guarantees that the first upstream-progressing solitary wave depicted in Fig. 1 is always fully formed at time $T$ throughout the iterative scheme.

In $\S 5.3$, we explain how the shape gradient (13) of the functional (3) is computed. Then, the $L^{2}$-constrained optimization problem (2) is replaced by an infinite sequence of unconstrained problems as in (17) thanks to the introduction of a Lagrange multiplier in $\S 5.4$. Finally, the Usawa-type procedure is discussed in $\S 5.5$, relying on the combination of two projected gradient methods. 


\subsection{Computation of the Shape Gradient of the Functional}

The techniques used to obtain (12) in Proposition 4.4 can be easily adapted to obtain the adjoint formulation of (6) with $\zeta=u$. Formally, calculations yield $\left(2 / c_{0}\right) \partial_{t} v+\left(3 / h_{0}\right) u \partial_{x} v+\left(h_{0}^{2} / 3\right) \partial_{x x x} v+2 u=0$ on $\mathbb{R} \times[0, T]$ and $v(\bullet, T)=0$. Then, the discretization is performed in the same way as (15) was obtained from (6), with the result:

$$
\begin{aligned}
\frac{2}{c_{0}} \frac{V_{i}^{n+1}-V_{i}^{n}}{\Delta t=-} & \frac{h_{0}^{2}}{6} \frac{\left(V_{i+2}^{n+1}-2 V_{i+1}^{n+1}+2 V_{i-1}^{n+1}-V_{i-2}^{n+1}\right)+\left(V_{i+2}^{n}-2 V_{i+1}^{n}+2 V_{i-1}^{n}-V_{i-2}^{n}\right)}{2 \Delta x^{3}} \\
& -\frac{3 U_{i}^{n+1}}{2 h_{0}} \frac{V_{i+1}^{n+1}-V_{i-1}^{n+1}}{2 \Delta x}-\frac{3 U_{i}^{n}}{2 h_{0}} \frac{V_{i+1}^{n}-V_{i-1}^{n}}{2 \Delta x}-\left(U_{i}^{n+1}+U_{i}^{n}\right) .
\end{aligned}
$$

As time is reversed, the system gets the matrix form $A^{n+1} V^{n}=B^{n+1}$. Using the trick of $\oint 5.2$, a filter $\mathfrak{f}$ is applied at each time step to ensure a smooth decreasing of $V^{n}$ to zero on $[-L,-L+\Delta L]$ and $[L-\Delta L, L]$, where $\left.\Delta L \in\right] 0, L[$ is the interval size on which the filter acts. We thus have:

$$
\left\{\begin{aligned}
V_{n}^{i} & =\mathfrak{f}\left(\mathbf{x}_{i}\right)\left[\left(A^{n+1}\right)^{-1} B^{n+1}\right]_{i} \\
\mathfrak{f}(x) & :=f(x)-\mathbf{1}_{[L-\Delta L, L]}(x)+\frac{1}{2}\left[1+\cos \left(\pi \frac{\Delta L+x-L}{\Delta L}\right)\right] \mathbf{1}_{[L-\Delta L, L]}(x) .
\end{aligned}\right.
$$

As illustrated in Fig. 3, the filter $\mathfrak{f}$ alters severely the numerical solution of (16) on the whole computational domain $[-L, L]$, whereas the filter $f$ only interfered on the interval $[-L,-L+\Delta L]$ where it acts. Fortunately, the effects of the filter are negligible on the computation of the shape gradient (13), which is the pertinent quantity for the numerical calculations. We can also observe that the corresponding shape gradients $\partial_{b} F$ and $\partial_{b} F_{\text {filter }}$ are almost proportional, i.e. $\partial_{b} F_{\text {filter }}=\left(1+\gamma_{\text {filter }}\right) \partial_{b} F$, where $\gamma_{\text {filter }}$ is small. Consequently, $\partial_{b} F$ and $\partial_{b} F_{\text {filter }}$ can be used indifferently in the optimization algorithm since the new bottom is evaluated by $b+\gamma \partial_{b} F_{\text {filter }}=b+\gamma\left(1+\gamma_{\text {filter }}\right) \partial_{b} F$, where $\gamma>0$ is small. Finally, note that the adjoint equation is a linearization of (6). In particular, similar results as those presented in (14) and Propositions B.2B.3 can be obtained for (16). To conclude, at each step of the optimization algorithm, (15) and (16) must be solved to compute (13), whose integral is approximated according to Simpson's rule.

\subsection{Description of the Optimization Algorithm}

Let $\mathcal{L}:(b, \lambda) \in L^{2}(\mathbb{R}, \mathbb{R}) \times[0,+\infty[\mapsto \mathcal{L}(b, \lambda) \in \mathbb{R}$ be the Lagrangian associated with $(2)$ and defined by $\mathcal{L}(b, \lambda):=F(b)+\lambda\left(M^{2}-\|b\|_{L^{2}(\mathbb{R}, \mathbb{R})}^{2}\right)$, then consider:

$$
G(\lambda):=\sup _{\substack{b \in L^{2}(\mathbb{R},[0,+\infty[) \\ \operatorname{supp} b \subseteq[-K, K]}} \mathcal{L}(b, \lambda)
$$



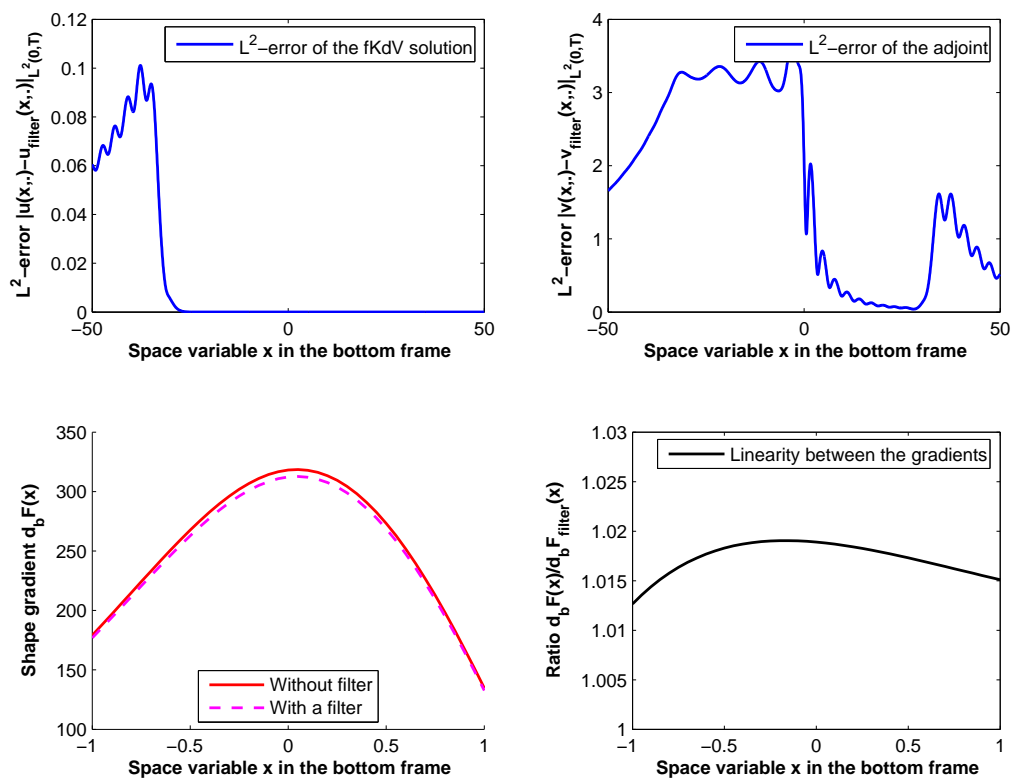

Fig. 3 Illustration of the effects on the solution due to the filters. The numerical simulation is implemented with the parameters: $\Delta x=0.05, L=50, \Delta L=20, \Delta t=0.1$, and $T=30$.

Usawa's method consists in relaxing the $L^{2}$-constraint by solving $\inf _{\lambda \geqslant 0} G(\lambda)$ instead of (2). In general, the two problems are not equivalent. We only have:

$$
\inf _{\lambda \geqslant 0} G(\lambda) \geqslant \sup _{b \in \mathcal{B}} F(b) .
$$

We were not able to prove analytically that equality holds in (18). Adapting some arguments of $\S 4$, we could however show the existence of $\lambda>0$ and a non-negative square-integrable map $b_{\lambda}$ such that $\mathcal{L}\left(b_{\lambda}, \lambda\right)=\inf _{\lambda>0} G(\lambda)$. If one could show the uniqueness of such map $b_{\lambda}$, then we could prove that $\left\|b_{\lambda}\right\|_{L^{2}(\mathbb{R}, \mathbb{R})}=M$, in which case (18) would be an equality and the global maximizer of (2) unique. Despite the lack of a theoretical proof, every single numerical simulation we performed solving $\inf _{\lambda \geqslant 0} G(\lambda)$ yielded a critical point $b^{\text {opt }}$ saturating the $L^{2}$-constraint to a very high order of precision $\left(\left\|b^{\text {opt }}\right\|_{L^{2}(\mathbb{R}, \mathbb{R})}=M\right.$ up to 8 digits). This strongly suggests the validity of our claim and justifies, at least numerically and a posteriori, the use of Usawa's method to our problem. We thus combine:

(i) a gradient method for the primal problem (17), in which the shape gradient of the Lagrangian is needed and given by $\partial_{b} \mathcal{L}(b, \lambda):=\partial_{b} F-2 \lambda b$;

(ii) a projected gradient method for the dual problem $\inf _{\lambda \geqslant 0} G(\lambda)$, using the explicit expression of the projector on $\left[0,+\infty\left[\right.\right.$, given by $P_{+}(\bullet):=\max (0, \bullet)$. 
Remark that (17) still contains the non-negativity and support constraint of the bottom. Therefore, we have to replace in (i) a gradient descent by a projected gradient method. The algorithm thus reads:

$$
\left\{\begin{array}{l}
b^{\text {new }}=P_{+}\left[b^{\text {old }}+\gamma \partial_{b} \mathcal{L}\left(b^{\text {old }}, \lambda^{\text {old }}\right)\right] \mathbf{1}_{[-K, K]} \\
\lambda^{\text {new }}=P_{+}\left[\lambda^{\text {old }}-\kappa\left(M^{2}-\left\|b^{\text {new }}\right\|_{L^{2}(\mathbb{R}, \mathbb{R})}^{2}\right)\right]
\end{array}\right.
$$

where $\gamma, \kappa>0$ are parameters to tune, and where $\mathbf{1}_{[-K, K]}$ equals one on $[-K, K]$ otherwise zero.

\subsection{Behaviour of the Usawa-Type Algorithm}

The general behaviour expected from the algorithm is depicted in Fig. 4. The initial bottom $b$ is a cosine-shaped profile satisfying the $L^{2}$-constraint. In particular, $\lambda=0$ and $\mathcal{L}(b, \lambda)=F(b)$. Hence, the initial deformation is only ruled by the functional. Then, it is expected from the algorithm to increase the bottom height because the wave elevation is very sensitive to it. However, after some iterations, the bottom will not satisfy the $L^{2}$-constraint and thus $\lambda>0$. The $L^{2}$-constraint begins to act in the deformation process in order to bring back the bottom into the admissible set.

Consequently, oscillations of the Lagrangian are expected around a saddle point corresponding to an equilibrium between the will of the functional and the constraints. The convergence of the algorithm mainly depends on how quickly the $L^{2}$-constraint intervenes in the optimization process, which is thus ruled by the parameter $\kappa$. As shown in Fig. 4, the convergence occurs if the parameter $\kappa$ is able to reduce progressively the oscillations observed on the evolution of all the characteristic parameters, such as the functional, the Lagrange multiplier and the amplitude of the solitary wave. An educated choice of $\kappa$ has been difficult to set up. Indeed, too small $\kappa$ mean a delay in the process of penalization of the functional whereas high values make the constraints immediately significant. In both cases, high oscillations will be observed. A typical value is $\kappa=30000$.

Then, we comment the choice of $\gamma$. The setting up of (17) via the gradient method (i) relies on the optimal local direction for steepest descent. Hence, the parameter $\gamma$ must be small since Taylor expansions around zero are involved. A typical value is $\gamma=10^{-4}$. Moreover, if $\gamma$ is too important, the new bottom will always leave the admissible set or violate the physical limit of the fKdV model. Finally, a stopping criterion $\iota>0$ is chosen small. It corresponds to the tolerance allowed on the precision of variables. The algorithm stops when the constraints are satisfied and $\left\|b^{\text {new }}-b^{\text {old }}\right\|_{L^{2}(\mathbb{R}, \mathbb{R})}<\iota$. A typical value is $\iota=\gamma 10^{-3}$. Indeed, we have $\left\|b^{\text {new }}-b^{\text {old }}\right\|_{L^{2}(\mathbb{R}, \mathbb{R})} \leqslant \gamma\left\|\mathcal{L}\left(b^{\text {old }}, \lambda^{\text {old }}\right)\right\|_{L^{2}(-K, K)}$ and numerics suggest that equality hold if $\gamma$ is chosen small enough. 

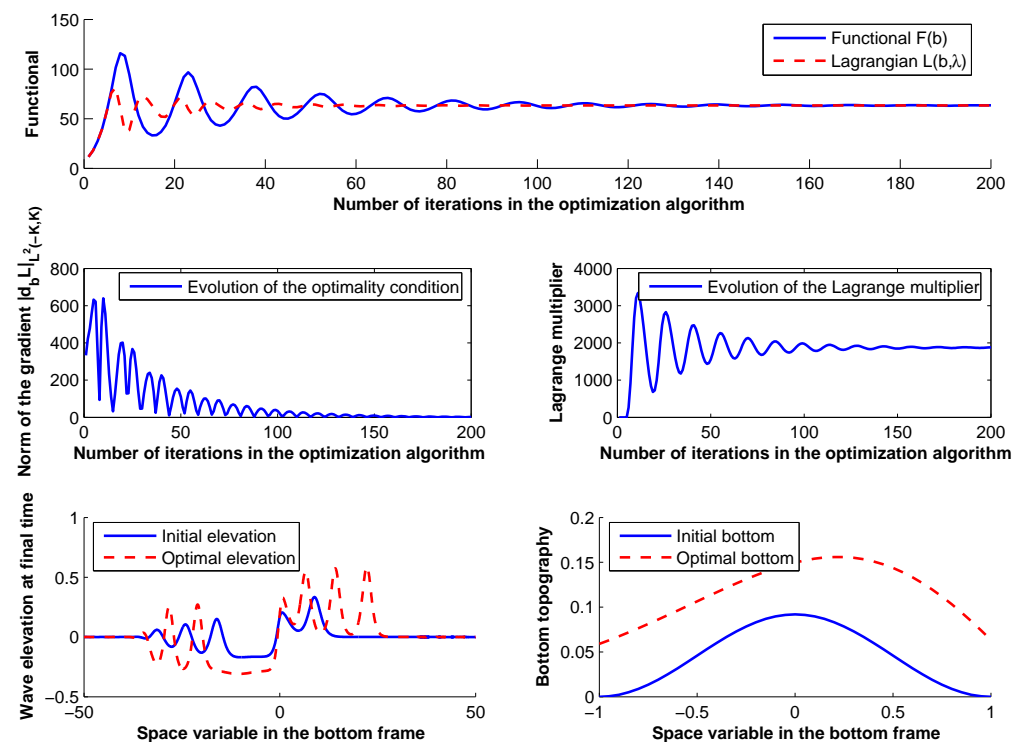

Fig. 4 Illustration of the algorithm convergence. The computational time is 20 minutes for the parameters: $M=\sqrt{0.03}$, supp $b \subseteq[-1,1], \Delta x=0.05, L=50, \Delta t=0.1, T=30$, $\kappa=30000, \gamma=0.00005$, and $\iota=\gamma 10^{-3}$.

\section{Numerical Results and Perspectives}

Figure 4 illustrates how, given an initial cosine-shaped profile satisfying the $L^{2}$-constraint, our method converges to an optimal bottom responsible for generating a leading solitary wave with almost a two-fold increase of amplitude with respect to the one generated by the initial bottom. Both initial bottom and optimal bottom have the same support restriction, but while the initial profile is continuous (when viewed as a function over the real line), the optimal one is not. A discontinuity can be observed at both ends of the support. The oscillating behaviour of the algorithm, and especially its difficulty to converge, is well present in this figure. This indicates that the soliton height is highly sensitive to the amplitude of the bottom topography and corroborates the findings in $[6]$.

We were interested in determining how this optimal shape depended on different prescribed initial conditions. As we know, for non-linear optimization problems endowed with non-convex functionals, several distinct critical points, local and global maxima can in general arise, preventing a gradient-based algorithm to converge to a global maximum. In such situations, we often rely on experience to provide an educated initial guess leading to a meaningful solution. Figure 5 displays the results obtained for different initial guesses for the 
bottom. A very unusual result that warrants further investigation can be observed here: regardless of the starting shapes, the optimization algorithm seems to converge to the same shape. This observation is quantified on the lower panel of Fig. 5, in which we present the distance between the optimal bottom $b_{\text {opt }}^{\text {ref }}$, resulting from an initial cosine shape and satisfying $\left\|b_{\text {opt }}^{\text {ref }}\right\|_{L^{2}(\mathbb{R}, \mathbb{R})}=M$ up to 8 digits, and the optimal bottoms obtained from the other five initial shapes. Discrepancies were found to be of order $10^{-7}$. Also included in the figure is the value of the first-order optimality condition, i.e. the shape gradient of the Lagrangian, for which obtained values are of order $10^{-4}$.
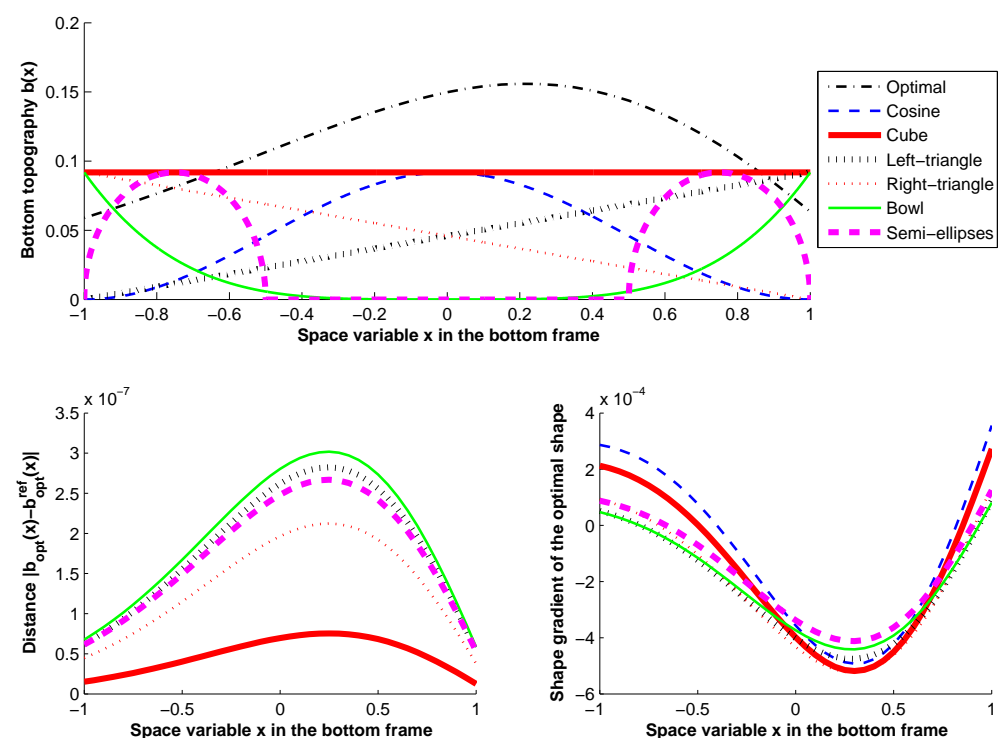

Fig. 5 The optimization algorithm is performed for various initial bottoms. Each optimal shape has been computed in 4 minutes for 90 iterations with the parameters: $M=\sqrt{0.03}$, $\operatorname{supp} b \subseteq[-1,1], \Delta x=0.05, L=50, \Delta L=20, \Delta t=0.1, T=30, \kappa=30000, \gamma=0.00025$, and $\iota=\gamma 10^{-3}$.

These are strong evidence of the uniqueness of a critical point for the optimization problem (2), which would imply that the shape obtained by the algorithm corresponds to a unique maximizer for (2), the optimal one described in Theorem 2.1, and gives further support to our claim made in $\S 5.4$ about the equality in (18).

We also investigate how the support constraint supp $b \subseteq\left[K_{-}, K_{+}\right]$influences the discontinuities of the optimal bottom that may manifest at the ends of the support considered. On the left panel of Fig. 6 , we set $K_{+}=1$ matching the right-end support of the initial condition, and display the optimal pro- 
files computed for $K_{-} \in\{-3.5,-2.5,-1.5,-0.5\}$. On the right panel, we set $K_{+}=1.5$ and consider the cases $K_{-} \in\{-3,-2,-1,0\}$. In all cases, the rightend discontinuity is present (although not at the same height). In contrast, the left-end discontinuity seems to strongly depend on the support restriction. Also, it is striking how resemblant the shapes are in the cases $K_{+}=1$ and $K_{+}=1.5$; in fact, almost indiscernible (of order $10^{-4}$ ) upon translation. It would be interesting to corroborate analytically these numerical observations, in particular that the optimal bottom is not $H^{1}$-regular over the real line.
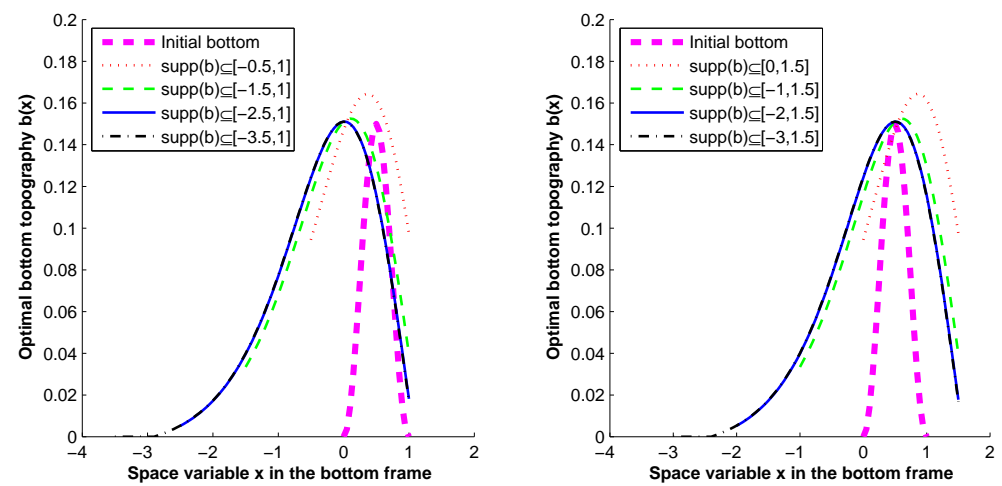

Fig. 6 Influence of the support restriction. For the same initial bottom, we determine how the optimal shape of the bottom varies with different supports. Each optimal shape has been computed in 4 minutes for 90 iterations with the same parameters as in Figure 5.

It is worth pointing out the shortcomings of an inviscid model as the one adopted here. However the gains in amplitude were rather important in the case displayed in Fig. 4, this only seems to be the case because the initial bottom is far from saturating the $L^{2}$-constraint. As a matter of fact, when in Fig. 5 the shape of the optimal bottom is compared to the highest admissible cube (rectangle to be more precise), the difference in amplitudes between the two leading waves is not that significant. Even if in reality that could be the case, it is not difficult to imagine that drag forces due to a cube would be much more important than those due to the optimal shape computed here (which reminds a section of an airfoil), hence with a far greater energy consumption associated to it. For a more realistic description of the problem, weak dissipative effects should be included in the model allowing in particular to characterize the adherence of the water on the bottom to better study the dependency between its shape and the amplitude of the generated wave. Despite the limitations, we are able to recover results which look very like those used in practice [2].

To conclude, we justify numerically the choice of the $L^{2}$-setting to study the optimization problem (2). Clearly, from a theoretical viewpoint, it would have been much easier to consider admissible bottoms $b \in H_{0}^{1}(]-K, K[,[0,+\infty[)$ 
satisfying $\|b\|_{H^{1}(\mathbb{R}, \mathbb{R})} \leqslant M$. However, in this case, our numerical approach provides an irregular perturbation $\partial_{b} \mathcal{L}(b, \lambda)=\partial_{b} F-2 \lambda\left(b-\partial_{x x} b\right) \in H^{-1}(\mathbb{R}, \mathbb{R})$. The computation immediately diverges, leading to errors and oscillations. The well-posedness of (4) is only proved at least for bottoms in $H^{-\frac{1}{2}}$, although it is actually not known whether this exponent is optimal [9, Remark 1.2]. Even for the $\mathrm{KdV}$ equation, the space $H^{-\frac{3}{4}}$ is critical for well-posedness [8]. Hence, there is few hope to get a stable program. In comparison, the great advantage of the $L^{2}$-setting is that the shape gradient of our Lagrangian remains a square integrable map so our numerical algorithm is stable with respect to the class of admissible bottoms.

\section{Conclusion}

In this article, the forced Korteweg-de Vries equation (4) is used to investigate how the shape of an underwater (steadily) moving bottom at a critical speed can affect the amplitude of the first upstream-progressing solitary wave. With the view of increasing the amplitude of such wave, under some practical constraints, we consider the non-linear optimal control problem (2) from a shape optimization perspective. This amounts to maximizing the total energy (3) of the system over the set (1) of non-negative square-integrable bottoms, with uniformly bounded $L^{2}$-norms and supports embedded in a given fixed compact set.

To the expense of a lengthy article, we have tried to exhibit here a rather thorough study of this design optimization problem. The paper contains indeed argued choices for the modelling, the proof of Theorem 2.1 stating the existence of a maximizer saturating the $L^{2}$-constraint, the derivation of a shape gradient, the setting up of a numerical Usawa-type algorithm yielding the desired optimal shape, an analysis of its efficiency and stability, and the behaviour of the optimal shape with respect to different initial guesses and support constraints. We believe our approach is original since it is motivated by very concrete applications (modelling the generation of artificial waves in surfing pools), numerical purposes (studying the design of an optimal shape with shape-gradient perturbations that are stable with respect to the set of admissible shapes), and theoretical considerations (controlling the Korteweg-de Vries equation by its low-regular second member on the whole real line).

Acknowledgements This work started in March 2010 under the original idea of E. Zuazua, who guided the author J. D. during an internship at the Basque Center for Applied Mathematics (Spain). J. D. gratefully acknowledges E. Zuazua for his support, dynamism, and guidance during this period, which now, looking back, feels like good old times. The work was then considerably improved while J. D. was finishing his Master degree at the Institut Elie Cartan de Lorraine (France), for which the author would like to acknowledge financial support and thank A. Henrot for the encouragement to pursue this study. R. B. acknowledges the support of Science Foundation Ireland under grant 12/IA/1683. Finally, we sincerely thank the Editors and anonymous Referees for their valuable comments, which helped us improve the quality of this manuscript. 


\section{Appendix A Proofs of the Main Results}

\section{A.1 Global Well-Posedness of the fKdV Equation}

Proof of Proposition 4.1 Assume $T>0$ is fixed and $b \in L^{2}(\mathbb{R}, \mathbb{R})$ is given. As a particular case of $\left[9\right.$, Theorem 1.2], with $\sigma=-1, f=-\frac{d b}{d x}$, and initial data $u_{0} \equiv 0$, there exists a solution $u_{b} \in C^{0}\left(0, T ; H^{2}(\mathbb{R}, \mathbb{R})\right)$ to the initial-value problem (4). Consider any other bottom $\mathfrak{b} \in L^{2}(\mathbb{R}, \mathbb{R})$, with corresponding solution $u_{\mathfrak{b}} \in C^{0}\left(0, T ; H^{2}(\mathbb{R}, \mathbb{R})\right)$, then set $\delta b=\mathfrak{b}-b$ and $\delta u=u_{\mathfrak{b}}-u_{b}$. Clearly, one has:

$$
\left\{\begin{array}{l}
\frac{\partial(\delta u)}{\partial t}+\frac{\partial}{\partial x}\left[\frac{(\delta u)^{2}}{2}+u_{b} \delta u+\frac{\partial^{2}(\delta u)}{\partial x^{2}}\right]=-\frac{\mathrm{d}(\delta b)}{\mathrm{d} x} \in H^{-1}(\mathbb{R}, \mathbb{R}), \\
\delta u(x, 0)=0, \\
x \in \mathbb{R} .
\end{array}\right.
$$

Although the partial derivatives in (19) have to be handled with care, since they are understood in distributional sense, we can still apply the integration-by-parts formula stated in $\left[15\right.$, Lemma 7.3] by considering the Gelfand triple $H^{1}(\mathbb{R}, \mathbb{R}) \subset L^{2}(\mathbb{R}, \mathbb{R}) \subset H^{-1}(\mathbb{R}, \mathbb{R})$ and the fact that we have $\delta u \in\left\{w \in L^{2}\left(0, T ; H^{1}(\mathbb{R}, \mathbb{R})\right): \partial_{t} w \in L^{2}\left(0, T ; H^{-1}(\mathbb{R}, \mathbb{R})\right)\right\}$. We thus obtain for any $t \in[0, T]$ :

$$
\|\delta u(\bullet, t)\|_{L^{2}(\mathbb{R}, \mathbb{R})}^{2}=\|\delta u(\bullet, 0)\|_{L^{2}(\mathbb{R}, \mathbb{R})}+2 \int_{0}^{t}\left\langle\partial_{t}(\delta u) \mid \delta u\right\rangle_{H^{-1}(\mathbb{R}, \mathbb{R}), H^{1}(\mathbb{R}, \mathbb{R})}(\bullet, s) \mathrm{d} s .
$$

We get $\left\langle\partial_{t}(\delta u) \mid \delta u\right\rangle_{H^{-1}(\mathbb{R}, \mathbb{R}), H^{1}(\mathbb{R}, \mathbb{R})}=\left\langle\frac{(\delta u)^{2}}{2}+u_{b} \delta u+\partial_{x x}(\delta u)+\delta b \mid \partial_{x}(\delta u)\right\rangle_{L^{2}(\mathbb{R}, \mathbb{R}), L^{2}(\mathbb{R}, \mathbb{R})}$ and $\delta u(\bullet, 0)=0$ by using $(19)$, from which it follows for any $t \in[0, T]$ :

$$
\|\delta u(\bullet, t)\|_{L^{2}(\mathbb{R}, \mathbb{R})}^{2}=2 \int_{0}^{t} \int_{\mathbb{R}} \delta b \frac{\partial(\delta u)}{\partial x} \mathrm{~d} x \mathrm{~d} s-\int_{0}^{t} \int_{\mathbb{R}}(\delta u)^{2} \frac{\partial u_{b}}{\partial x} \mathrm{~d} x \mathrm{~d} s .
$$

We may then write, by introducing $C:=\max \left(\left\|u_{b}\right\|_{C^{0}\left(0, T ; H^{2}(\mathbb{R}, \mathbb{R})\right)},\left\|u_{\mathfrak{b}}\right\|_{C^{0}\left(0, T ; H^{2}(\mathbb{R}, \mathbb{R})\right)}\right)$, which is a finite constant:

$$
\forall t \in[0, T], \quad\|\delta u(\bullet, t)\|_{L^{2}(\mathbb{R}, \mathbb{R})}^{2} \leqslant 4 C T\|\delta b\|_{L^{2}(\mathbb{R}, \mathbb{R})}+C \int_{0}^{t}\|\delta u(\bullet, s)\|_{L^{2}(\mathbb{R}, \mathbb{R})}^{2} \mathrm{~d} s .
$$

Since $t \in[0, T] \mapsto\|\delta u(\bullet, t)\|_{L^{2}(\mathbb{R}, \mathbb{R})} \in \mathbb{R}$ is a continuous function [15, Lemma 7.3], we can apply Grönwall's Lemma, which yields

$$
\forall t \in[0, T], \quad\left\|u_{\mathfrak{b}}(\bullet, t)-u_{b}(\bullet, t)\right\|_{L^{2}(\mathbb{R}, \mathbb{R})}^{2} \leqslant 4 C T e^{C t}\|\mathfrak{b}-b\|_{L^{2}(\mathbb{R}, \mathbb{R})} .
$$

In particular, the uniqueness of solution to the initial-value problem (4) follows and the functional $F: b \mapsto F(b)$ given by (3) is a well defined application from $L^{2}(\mathbb{R}, \mathbb{R})$ into $\mathbb{R}$.

\section{A.2 Explicit A Priori Estimates}

Proposition A.1 Let $T>0$ and $b \in H^{\infty}(\mathbb{R}, \mathbb{R}):=\cap_{s \geqslant 0} H^{s}(\mathbb{R}, \mathbb{R})$. Then, there exist three polynomials $P_{0}, P_{1}, P_{2}$ in two variables with (non-negative) constant coefficients, such that the following estimates hold for the unique solution $u_{b} \in C^{\infty}\left(0, T ; H^{\infty}(\mathbb{R}, \mathbb{R})\right)$ to the initial-value problem (4):
(i) $\sup _{t \in[0, T]}\left\|u_{b}(\bullet, t)\right\|_{L^{2}(\mathbb{R}, \mathbb{R})} \leqslant P_{0}\left(T,\|b\|_{L^{2}(\mathbb{R}, \mathbb{R})}\right)$,
(ii) $\sup _{t \in[0, T]}\left\|\partial_{x} u_{b}(\bullet, t)\right\|_{L^{2}(\mathbb{R}, \mathbb{R})} \leqslant P_{1}\left(T,\|b\|_{L^{2}(\mathbb{R}, \mathbb{R})}\right)$,
(iii) $\sup _{t \in[0, T]}\left\|\partial_{x x} u_{b}(\bullet, t)\right\|_{L^{2}(\mathbb{R}, \mathbb{R})} \leqslant e^{T\left(1+\frac{1}{3}\|b\|_{L^{2}(\mathbb{R}, \mathbb{R})}^{2}\right)} P_{2}\left(T,\|b\|_{L^{2}(\mathbb{R}, \mathbb{R})}\right)$. 
Proof Let $T>0$ and $b \in H^{\infty}(\mathbb{R}, \mathbb{R}):=\cap_{s} \geqslant 0 H^{s}(\mathbb{R}, \mathbb{R})$. Lemmas 3.1 and 3.2 imply the existence of a unique smooth solution $u_{b} \in C^{\infty}\left(0, T ; H^{\infty}(\mathbb{R}, \mathbb{R})\right)$ of $(4)$. Since $b \in L^{2}(\mathbb{R}, \mathbb{R})$, we may apply [9, Proposition 3.1] with final time $T+1(>1), \sigma=-1, f=-\frac{d b}{d x}$, and homogeneous initial data $u_{0} \equiv 0$, to establish the inequality:

$\sup _{t \in[0, T]}\left\|u_{b}(\bullet, t)\right\|_{L^{2}(\mathbb{R}, \mathbb{R})} \leqslant \sup _{t \in[0, T+1]}\left\|u_{b}(\bullet, t)\right\|_{L^{2}(\mathbb{R}, \mathbb{R})} \leqslant C\left(1+(1+T)^{3}\left\|\partial_{x} b\right\|_{H^{-1}(\mathbb{R}, \mathbb{R})}^{3}\right)$,

for some positive constant $C$, which does not depend on $T, b$, or $u_{b}$. This proves assertion (i) with $P_{0}(x, y):=C\left(1+(1+x)^{3} y^{3}\right)$, using here the fact that $\left\|\partial_{x} b\right\|_{H^{-1}(\mathbb{R}, \mathbb{R})} \leqslant\|b\|_{L^{2}(\mathbb{R}, \mathbb{R})}$. We now exploit the Hamiltonian structure of equation (4) (see [22]). Although energy is not conserved, as already pointed out, an extra conserved quantity is available for the fKdV equation, which is in fact a Hamiltonian for the system. Let $H$ be such Hamiltonian. Then,

$$
\forall t \in[0, T], \quad H(t):=\int_{\mathbb{R}}\left[\left(\frac{\partial u_{b}}{\partial x}\right)^{2}-\frac{1}{3} u_{b}^{3}-2 b u_{b}\right] \mathrm{d} x=0 .
$$

The Cauchy-Schwarz inequality and $\|g\|_{L^{\infty}(\mathbb{R}, \mathbb{R})}^{2} \leqslant 2\|g\|_{L^{2}(\mathbb{R}, \mathbb{R})}\left\|\partial_{x} g\right\|_{L^{2}(\mathbb{R}, \mathbb{R})} \leqslant\|g\|_{H^{1}(\mathbb{R}, \mathbb{R})}^{2}$ valid for any $g \in H^{1}(\mathbb{R}, \mathbb{R})$ are combined with the well-known inequalities $2 x y \leqslant x^{2}+y^{2}$ and $\sqrt{x+y} \leqslant \sqrt{x}+\sqrt{y}$, valid for any $x, y \geqslant 0$, so that one can deduce from (20):

$$
\left\|\partial_{x} u_{b}(\bullet, t)\right\|_{L^{2}(\mathbb{R}, \mathbb{R})} \leqslant \sqrt{\frac{7}{5}}\left(\left\|u_{b}(\bullet, t)\right\|_{L^{2}(\mathbb{R}, \mathbb{R})}+\left\|u_{b}(\bullet, t)\right\|_{L^{2}(\mathbb{R}, \mathbb{R})}^{2}+\|b\|_{L^{2}(\mathbb{R}, \mathbb{R})}\right) .
$$

This proves the estimate $(i i)$ of our Proposition A.1 with $P_{1}(x, y):=2\left[y+P_{0}(x, y)+P_{0}^{2}(x, y)\right]$. Finally, the same method is used to determine $P_{2}$. We first show for any $t \in[0, T]$ :

$$
\frac{\mathrm{d}}{\mathrm{d} t} \int_{\mathbb{R}}\left[\left(\partial_{x x} u_{b}\right)^{2}+2 b \partial_{x x} u_{b}-\frac{5}{3} u_{b}\left(\partial_{x} u_{b}\right)^{2}+\frac{2}{3} b u_{b}^{2}+\frac{5}{36} u_{b}^{4}\right] \mathrm{d} x=\frac{2}{3} \int_{\mathbb{R}} b I \partial_{x} u_{b} \mathrm{~d} x,
$$

with $I:=\partial_{x x} u_{b}+\frac{1}{2} u_{b}^{2}+b$. Notice that both sides of (21) depend only on time $t$. Denote by $G(t)$ the left-hand side of the equation. Straightforward calculations lead to:

$$
\begin{aligned}
G(t) & =\int_{\mathbb{R}} 2 \partial_{x t} u_{b} \underbrace{\left[-\partial_{x x x} u_{b}-b_{x}\right]}_{=\partial_{t} u_{b}+u_{b} \partial_{x} u_{b}}-\frac{10}{3} u_{b} \partial_{x} u_{b} \partial_{x t} u_{b}-\frac{5}{3} \partial_{t} u_{b}\left(\partial_{x} u_{b}\right)^{2}+\frac{4}{3} b u_{b} \partial_{t} u_{b} \begin{array}{r}
5 \\
+\frac{5}{9} u_{b}^{3} \partial_{t} u_{b}
\end{array} \\
& =\int_{\mathbb{R}} \frac{4}{3} u_{b} \underbrace{\partial_{t} u_{b}}_{=-I_{x}} \underbrace{\left[\partial_{x x} u_{b}+\frac{u_{b}^{2}}{2}+b\right]}_{:=I}-\frac{1}{3} \underbrace{\partial_{t} u_{b}}_{=-I_{x}}\left(\partial_{x} u_{b}\right)^{2}-\frac{1}{9} u_{b}^{3} \underbrace{\partial_{t} u_{b}}_{=-I_{x}}=\int_{\mathbb{R}} \frac{2}{3} \partial_{x} u_{b} I b .
\end{aligned}
$$

Integrating equality $(21)$ on $[0, t]$ for any $t \in[0, T]$, following the same strategy above yields

$$
\left\|\partial_{x x} u_{b}(\bullet, t)\right\|_{L^{2}(\mathbb{R}, \mathbb{R})}^{2} \leqslant 2\left(1+\frac{\|b\|_{L^{2}(\mathbb{R}, \mathbb{R})}^{2}}{3}\right) \int_{0}^{t}\left\|\partial_{x x} u_{b}(\bullet, s)\right\|_{L^{2}(\mathbb{R}, \mathbb{R})}^{2} \mathrm{~d} s+P_{01}\left(T,\|b\|_{L^{2}(\mathbb{R}, \mathbb{R})}\right)
$$

where we have set

$$
\begin{aligned}
P_{01}(x, y):= & 2 x\left[y^{2}\left(1+\frac{P_{1}(x, y)^{2}}{3}\right)+\frac{P_{0}(x, y)^{2}}{4}\left(P_{0}(x, y)^{2}+P_{1}(x, y)^{2}\right)\right] \\
+ & 2\left[2 y^{2}+\frac{5 P_{1}(x, y)}{6}\left(P_{0}(x, y)^{2}+2 P_{1}(x, y)^{2}\right)+\frac{y}{3}\left(2 P_{0}(x, y)^{2}+P_{1}(x, y)^{2}\right)\right. \\
& \left.+\frac{5 P_{0}(x, y)^{2}}{36}\left(P_{0}(x, y)^{2}+P_{1}(x, y)^{2}\right)\right] .
\end{aligned}
$$

Consequently, we apply Grönwall's Lemma to the last inequality above, from which the assertion (iii) follows by setting $P_{2}(x, y):=1+P_{01}(x, y)$, and concluding the proof. 
Proof of Proposition 4.2 Let $T>0$ and $b \in L^{2}(\mathbb{R}, \mathbb{R})$. First, according to Proposition 4.1, we can consider the unique solution $u_{b} \in C^{0}\left(0, T ; H^{2}(\mathbb{R}, \mathbb{R})\right)$ of (4). Moreover, by density, a sequence $\left(b_{n}\right)_{n \in \mathbb{N}}$ of smooth maps with compact support is strongly converging to $b$ in $L^{2}(\mathbb{R}, \mathbb{R})$. In addition, Proposition A.1 ensures that the sequence $\left(u_{b_{n}}\right)_{n \in \mathbb{N}}$ of associated smooth maps also satisfies (4) and the a priori estimates for any $n \in \mathbb{N}$. We deduce from the quantitative estimate of Proposition 4.1 the strong convergence of $\left(u_{b_{n}}\right)_{n \in \mathbb{N}}$ to $u_{b}$ in $C^{0}\left(0, T ; L^{2}(\mathbb{R}, \mathbb{R})\right)$. In particular, we can correctly let $n \rightarrow+\infty$ in the inequality $(i)$ of Proposition A.1 applied to $\left(u_{b_{n}}, b_{n}\right)$ in order to get $\left\|u_{b}\right\|_{C^{0}\left(0, T ; L^{2}(\mathbb{R}, \mathbb{R})\right)} \leqslant P_{0}\left(T,\|b\|_{L^{2}(\mathbb{R}, \mathbb{R})}\right)$.

Then, let $t \in[0, T]$ fixed. Since $\left(b_{n}\right)_{n \in \mathbb{N}}$ is bounded, the sequence $\left(\partial_{x} u_{b_{n}}(\bullet, t)\right)_{n \in \mathbb{N}}$ is uniformly bounded in $H^{1}(\mathbb{R}, \mathbb{R})$. Consequently, there exists a subsequence that weakly converges in $H^{1}(\mathbb{R}, \mathbb{R})$ to a certain map, which has to be $\partial_{x} u_{b}(\bullet, t)$ by considering the convergence in distributional sense. We emphasize the fact that here the subsequence depends on the time variable so it is denoted by $\left(\partial_{x} u_{b_{n(t)}}\right)_{n \in \mathbb{N}}$. Considering the lower-semicontinuity of the norm with respect to the weak convergence, we obtain for any $t \in[0, T]$ :

$$
\begin{array}{r}
\left\|\partial_{x} u_{b}(\bullet, t)\right\|_{H^{1}(\mathbb{R}, \mathbb{R})} \leqslant \liminf _{n \in \mathbb{N}}\left\|\partial_{x} u_{b_{n(t)}}(\bullet, t)\right\|_{H^{1}(\mathbb{R}, \mathbb{R})} \leqslant P_{2}\left(T,\|b\|_{L^{2}(\mathbb{R}, \mathbb{R})}\right) e^{T+\frac{T}{3}\|b\|_{L^{2}(\mathbb{R}, \mathbb{R})}^{2}} \\
+P_{1}\left(T,\|b\|_{L^{2}(\mathbb{R}, \mathbb{R})}\right) .
\end{array}
$$

Hence, the inequality of Proposition 4.2 holds with $\left(b, u_{b}\right)$, concluding the proof.

\section{A.3 Existence of an Optimal Bottom}

Proof of Proposition 4.3 The proof is very similar to the one of Proposition 4.1. Let $T>0$, $K>0$ and $b \in L^{2}(\mathbb{R}, \mathbb{R})$ with support included in $[-K, K]$. From Proposition 4.1 , the initial-value problem (4) has a unique solution $u_{b} \in C^{0}\left(0, T ; H^{2}(\mathbb{R}, \mathbb{R})\right)$. Consider now any sequence $\left(b_{n}\right)_{n \in \mathbb{N}}$ of square-integrable maps with supports all included in $[-K, K]$ that is weakly converging to $b$ in $L^{2}(\mathbb{R}, \mathbb{R})$. In particular, such a sequence is bounded so Propositions 4.1-4.2 ensure that the sequence $\left(u_{b_{n}}\right)_{n \in \mathbb{N}}$ of associated maps satisfies (4) and the a priori estimate for any $n \in \mathbb{N}$. We deduce that $u_{b}$ and $\left(u_{b_{n}}\right)_{n \in \mathbb{N}}$ are uniformly bounded in $C^{0}\left(0, T ; H^{2}(\mathbb{R}, \mathbb{R})\right)$. First, we consider the two following compact embeddings: $H^{2}(]-K, K[, \mathbb{R}) \subset H^{1}(]-K, K[, \mathbb{R}) \subset H^{-1}(]-K, K[, \mathbb{R})$. We can apply the Aubin-LionsSimon Lemma [14, Section 8 Corollary 4] to obtain that the following one is also compact:

$$
\begin{aligned}
W:=\left\{w \in L^{\infty}\left(0, T ; H^{2}(]-K, K[, \mathbb{R})\right): \partial_{t} w \in L^{\infty}(0, T\right. & \left.\left.; H^{-1}(]-K, K[, \mathbb{R})\right)\right\} \\
\hookrightarrow & C^{0}\left(0, T ; H^{1}(]-K, K[, \mathbb{R})\right) .
\end{aligned}
$$

From the foregoing and (4), $\left\|u_{b_{n}}\right\|_{L^{\infty}\left(0, T ; H^{2}(]-K, K[, \mathbb{R})\right)}+\left\|\partial_{t} u_{b_{n}}\right\|_{L^{\infty}\left(0, T ; H^{-1}(]-K, K[, \mathbb{R})\right)}$ is uniformly bounded i.e. $\left(u_{b_{n}}\right)_{n \in \mathbb{N}}$ is uniformly bounded in $W$. We deduce that there exists $u_{K} \in C^{0}\left(0, T ; H^{1}(]-K, K[, \mathbb{R})\right)$ and a subsequence $\left(u_{b_{n^{\prime}}}\right)_{n \in \mathbb{N}}$ that is strongly converging to $u_{K}$ in $C^{0}\left(0, T ; H^{1}(]-K, K[, \mathbb{R})\right)$. Then, let $n \in \mathbb{N}$. We introduce the quantities $\delta b=b_{n^{\prime}}-b$ and $\delta u=u_{b_{n^{\prime}}}-u_{b}$. One can check they satisfy the initial-value problem (19). We emphasize again the fact that the partial derivatives in (19) have to be handled with care since these are understood in a distributional sense. But we can still apply the integration-by-parts formula of $[15$, Lemma 7.3$]$ by considering the Gelfand triple $H^{1}(\mathbb{R}, \mathbb{R}) \subset L^{2}(\mathbb{R}, \mathbb{R}) \subset H^{-1}(\mathbb{R}, \mathbb{R})$ and the fact that $\delta u \in\left\{w \in L^{2}\left(0, T ; H^{1}(\mathbb{R}, \mathbb{R})\right): \partial_{t} w \in L^{2}\left(0, T ; H^{-1}(\mathbb{R}, \mathbb{R})\right)\right\}$. Following the same calculations than we did in the proof of Proposition 4.1, we get for any $t \in[0, T]$ :

$$
\|\delta u(\bullet, t)\|_{L^{2}(\mathbb{R}, \mathbb{R})}^{2}=2 \int_{0}^{t} \int_{\mathbb{R}} \delta b \frac{\partial(\delta u)}{\partial x} \mathrm{~d} x \mathrm{~d} s-\int_{0}^{t} \int_{\mathbb{R}}(\delta u)^{2} \frac{\partial u_{b}}{\partial x} \mathrm{~d} x \mathrm{~d} s .
$$

Finally, we use the Cauchy-Schwarz inequality and the fact that all the supports of the considered bottoms are included in $[-K, K]$. We thus get for any $t \in[0, T]$ :

$$
\begin{aligned}
\left\|\left(u_{b_{n^{\prime}}}-u_{b}\right)(\bullet, t)\right\|_{L^{2}(\mathbb{R}, \mathbb{R})}^{2} \leqslant & 2 \int_{0}^{T}\left|\int_{-K}^{K}\left(b_{n^{\prime}}-b\right)(x)\left[\frac{\partial u_{b_{n^{\prime}}}}{\partial x}-\frac{\partial u_{b}}{\partial x}\right](x, s) \mathrm{d} x\right| \mathrm{d} s \\
& +\left\|u_{b}\right\|_{C^{0}\left(0, T ; H^{2}(\mathbb{R}, \mathbb{R})\right)} \int_{0}^{t}\left\|\left(u_{b_{n^{\prime}}}-u_{b}\right)(\bullet, s)\right\|_{L^{2}(\mathbb{R}, \mathbb{R})}^{2} \mathrm{~d} s .
\end{aligned}
$$


Since $t \in[0, T] \mapsto\|\delta u(\bullet, t)\|_{L^{2}(\mathbb{R}, \mathbb{R})} \in \mathbb{R}$ is a continuous function [15, Lemma 7.3], we can apply Grönwall's Lemma and we obtain:

$$
\left\|u_{b_{n^{\prime}}}-u_{b}\right\|_{C^{0}\left(0, T ; L^{2}(\mathbb{R}, \mathbb{R})\right)}^{2} \leqslant C \int_{0}^{T}\left|\int_{-K}^{K}\left(b_{n^{\prime}}-b\right)(x)\left[\frac{\partial u_{b_{n^{\prime}}}}{\partial x}-\frac{\partial u_{b}}{\partial x}\right](x, s) \mathrm{d} x\right| \mathrm{d} s,
$$

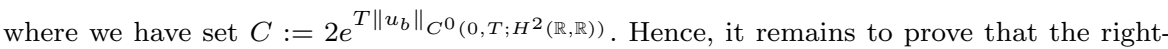
member of the above inequality converges to zero as $n \rightarrow+\infty$. For this purpose, let us now introduce the integrand $R_{n}: t \mapsto \int_{-K}^{K} \delta b(x) \partial_{x}(\delta u)(x, t) \mathrm{d} x$. Since $b_{n^{\prime}}$ converges weakly to $b$ in $L^{2}(]-K, K[, \mathbb{R})$ and $u_{b_{n}}$, strongly to $u_{K}$ in $C^{0}\left(0, T ; H^{1}(]-K, K[, \mathbb{R})\right)$, we get that $R_{n}(t)$ converges to zero for any $t \in[0, T]$. Moreover, the a priori estimate of Proposition 4.2 ensures that $R_{n}(t)$ is uniformly bounded. Hence, Lebesgue's Dominated Convergence Theorem applies and $\int_{0}^{T}\left|R_{n}(t)\right| \mathrm{d} t \rightarrow 0$ as $n \rightarrow+\infty$. One concludes from the last inequality that $\left(u_{b_{n^{\prime}}}\right)_{n \in \mathbb{N}}$ strongly converges to $u_{b}$ in $C^{0}\left(0, T ; L^{2}(\mathbb{R}, \mathbb{R})\right)$. We also have proved the uniqueness of the limit for any other converging subsequence. Recalling that $\left(u_{b_{n}}\right)_{n \in \mathbb{N}}$ is uniformly bounded, we deduce that the whole sequence converges to $u_{b}$.

Proof of Theorem 2.1 Combining Lemma 4.1 and Proposition 4.3, it is possible to extract from any maximizing sequence of $(2)$ a subsequence $\left(b_{n^{\prime}}\right)_{n \in \mathbb{N}}$ that is weakly converging in $L^{2}(\mathbb{R}, \mathbb{R})$ to a certain $b^{*} \in \mathcal{B}$, and such that $u_{b_{n^{\prime}}} \rightarrow u_{b^{*}}$ in $C^{0}\left(0, T ; L^{2}(\mathbb{R}, \mathbb{R})\right)$. From the continuity of the embedding $C^{0}\left(0, T ; L^{2}(\mathbb{R}, \mathbb{R})\right) \subset L^{2}\left(0, T ; L^{2}(\mathbb{R}, \mathbb{R})\right)$, we deduce that $F\left(b^{*}\right)=\lim _{n \rightarrow+\infty} F\left(b_{n^{\prime}}\right)=\sup _{b \in \mathcal{B}} F(b)$ with $b^{*} \in \mathcal{B}$ so the supremum is a maximum and problem (2) has a global maximizer. To conclude the proof, it remains to show that such a maximizer saturates the $L^{2}$-constraint, which is proved as in Proposition B.4. Indeed, if it not the case, then choose $\left.\theta \in] 1,\left(M /\left\|b^{\text {opt }}\right\|_{L^{2}(\mathbb{R}, \mathbb{R})}\right)^{2 / 7}\right]$ and the bottom $b_{\theta}^{\text {opt }}$ of Lemma B.1 is admissible. One can check that $F\left(b_{\theta}^{\mathrm{opt}}\right) \geqslant F\left(b^{\mathrm{opt}}\right)$ and the optimality of $b^{\text {opt }}$ yields to $u_{b_{\text {opt }}}=0$ on $\left[T, \theta^{3} T\right]$. Considering now $(4)$, we obtain $\partial_{x} b^{\text {opt }}=0$ so $u_{b^{\text {opt }}}=0$ also on $[0, T]$ and $F\left(b^{\mathrm{opt}}\right)=0$, which is a contradiction.

\section{A.4 Fréchet Differentiability of the Functional}

Proof of Proposition 4.4 Formally speaking, if $v_{b}$ is a solution of (12), then the equation satisfied by $\partial_{x} v_{b}$ is already studied in [7]. However, we need to specify a bit the existence result because it is stated in terms of the so-called Bourgain space $Y_{s, \beta}$ (see [7, §2] for details). First, we apply [9, Proposition 2.1] with $\sigma=-1, f=-\partial_{x} b, u_{0} \equiv 0, s=\sigma+3=2$ and $\beta=\varepsilon+\frac{1}{2}$, where $\varepsilon>0$ is chosen small enough. This local existence result combined with standard global arguments [7, Proposition 5.1] establishes that the unique solution $u_{b}$ of (4) is the $[0, T]$-restriction of a map $U_{b} \in Y_{2, \varepsilon+1 / 2}$.

Then, we introduce new variables $(\xi, \tau):=(-x, T-t)$ to transform (12) into an initialvalue problem. We set $U(\xi, \tau):=U_{b}(-x, T-t)$ and we still have $U \in Y_{2, \varepsilon+1 / 2} \subset Y_{1, \varepsilon+1 / 2}$ but we also get $\partial_{\xi} U \in Y_{1, \varepsilon+1 / 2}[7$, above Theorem 5.5]. We can now apply [7, Theorem 2.6] with $s=1, \beta=\varepsilon+\frac{1}{2}$, and $f=2 \partial_{\xi} U$. We deduce that there exists a unique solution $W \in Y_{1, \varepsilon+1 / 2}$ satisfying $W(\bullet, 0)=0$ and $\partial_{\tau} W+\partial_{\xi}(U W)+\partial_{\xi \xi \xi} W=2 \partial_{\xi} U$ on $\mathbb{R} \times[0, T]$.

Finally, it remains to get back to $(12)$. For this purpose, we consider the $[0, T]$-restrictions $u \in C^{0}\left(0, T ; H^{2}(\mathbb{R}, \mathbb{R})\right), w \in C^{0}\left(0, T ; H^{1}(\mathbb{R}, \mathbb{R})\right)$ of the maps $U$ and $W[7$, Lemma 2.3]. Using the equations they satisfy, we get $u(2-w) \in W^{1,1}\left(0, T ; H^{-2}(\mathbb{R}, \mathbb{R})\right)$. From standard linear semi-group theory $[7$, Section $1 \S \mathrm{III}]$, there exists a unique function $v \in C^{0}\left(0, T ; H^{1}(\mathbb{R}, \mathbb{R})\right)$ satisfying $v(\bullet, 0)=0$ and the Airy equation $\partial_{\tau} v+\partial_{\xi \xi \xi} v=u(2-w)$ on $\mathbb{R} \times[0, T]$. Looking at the equation satisfied by $w-\partial_{x} v$, we deduce that $\partial_{x} v=w$. In particular, we obtain $v \in C^{0}\left(0, T ; H^{2}(\mathbb{R}, \mathbb{R})\right.$ and getting back to the original variables $(x, t):=(-\xi, T-\tau)$, one can check that the map $v_{b}(x, t):=v(\xi, \tau)$ is a global solution of $(12)$ in $C^{0}\left(0, T ; H^{2}(\mathbb{R}, \mathbb{R})\right)$.

At last, we prove such a solution is unique. Consider two maps of $C^{0}\left(0, T ; H^{2}(\mathbb{R}, \mathbb{R})\right)$ solving (12), denoted $v_{1}$ and $v_{2}$, and introduce the quantity $\delta v:=v_{1}-v_{2}$. From the linearity of (12), one can check that $\delta v$ satisfies $\delta v(\bullet, T)=0$ and $\partial_{t}(\delta v)+u_{b} \partial_{x}(\delta v)+\partial_{x x x}(\delta v)=0$. This 
last equality is understood in distributional sense but we can still apply the integration-byparts formula of $\left[15\right.$, Lemma 7.3] with the Gelfand triple $H^{1}(\mathbb{R}, \mathbb{R}) \subset L^{2}(\mathbb{R}, \mathbb{R}) \subset H^{-1}(\mathbb{R}, \mathbb{R})$ and the fact that $\delta v \in\left\{w \in L^{2}\left(0, T ; H^{1}(\mathbb{R}, \mathbb{R})\right): \partial_{t} w \in L^{2}\left(0, T ; H^{-1}(\mathbb{R}, \mathbb{R})\right)\right\}$. Proceeding as below (19), we get:

$$
\forall t \in[0, T], \quad\|\delta v(\bullet, t)\|_{L^{2}(\mathbb{R}, \mathbb{R})}^{2} \leqslant\left\|\partial_{x} u_{b}\right\|_{C^{0}\left(0, T ; H^{1}(\mathbb{R}, \mathbb{R})\right)} \int_{t}^{T}\|\delta v(\bullet, s)\|_{\left.L^{2}(\mathbb{R}, \mathbb{R})\right)}^{2} \mathrm{~d} s .
$$

It follows from the continuity of the map $t \in[0, T] \mapsto\|\delta v(\bullet, t)\|_{L^{2}(\mathbb{R}, \mathbb{R})} \in \mathbb{R}[15$, Lemma 7.3] and Grönwall's Lemma that $\delta v \equiv 0$ on $[0, T] \times \mathbb{R}$ i.e. $v_{1}=v_{2}$. To conclude the proof, there exists a unique global solution $v_{b} \in C^{0}\left(0, T ; H^{2}(\mathbb{R}, \mathbb{R})\right)$ satisfying (12).

Proof of Proposition 4.5 Let $T>0$ and $(b, h) \in L^{2}(\mathbb{R}, \mathbb{R}) \times L^{2}(\mathbb{R}, \mathbb{R})$. From Proposition 4.1, there exists two associated global solutions $u_{b}$ and $u_{b+h}$ in $C^{0}\left(0, T ; H^{2}(\mathbb{R}, \mathbb{R})\right)$ such that $\left(b, u_{b}\right)$ and $\left(b+h, u_{b+h}\right)$ satisfy (4). Introducing again the quantities $\delta u:=u_{b+h}-u_{b}$ and $\delta b:=(b+h)-b=h$, one can check $(\delta b, \delta u)$ satisfies $(19)$, which has to be understood in a distributional sense. Still, we can apply the integration-by-parts formula of [15, Lemma 7.3] with the Gelfand triple $H^{1}(\mathbb{R}, \mathbb{R}) \subset L^{2}(\mathbb{R}, \mathbb{R}) \subset H^{-1}(\mathbb{R}, \mathbb{R})$ and the fact that $\delta u$ belongs to $\left\{w \in L^{2}\left(0, T ; H^{1}(\mathbb{R}, \mathbb{R})\right): \partial_{t} w \in L^{2}\left(0, T ; H^{-1}(\mathbb{R}, \mathbb{R})\right)\right\}$. Proceeding as below (19), we get:

$$
\forall t \in[0, T], \quad\|\delta u(\bullet, t)\|_{L^{2}(\mathbb{R}, \mathbb{R})}^{2}=2 \int_{0}^{t} \int_{\mathbb{R}} \delta b \frac{\partial(\delta u)}{\partial x} \mathrm{~d} x \mathrm{~d} s-\int_{0}^{t} \int_{\mathbb{R}}(\delta u)^{2} \frac{\partial u_{b}}{\partial x} \mathrm{~d} x \mathrm{~d} s .
$$

Using the Cauchy-Schwarz inequality, we obtain for any $t \in[0, T]$ :

$$
\|\delta u(\bullet, t)\|_{L^{2}(\mathbb{R}, \mathbb{R})}^{2} \leqslant C \int_{0}^{t}\|\delta u(\bullet, s)\|_{L^{2}(\mathbb{R}, \mathbb{R})}^{2} \mathrm{~d} s+2 T\|h\|_{L^{2}(\mathbb{R}, \mathbb{R})}\|\delta u\|_{C^{0}\left(0, T, H^{1}(\mathbb{R}, \mathbb{R})\right.},
$$

where we have set $C:=\left\|u_{b}\right\|_{C^{0}\left(0, T, H^{2}(\mathbb{R}, \mathbb{R})\right.}$. We can now apply Grönwall's Lemma to the map $t \in[0, T] \mapsto\|\delta u(\bullet, t)\|_{L^{2}(\mathbb{R}, \mathbb{R})} \in \mathbb{R}$, which is continuous [15, Lemma 7.3], and it comes:

$$
\left\|u_{b+h}-u_{b}\right\|_{C^{0}\left(0, T ; L^{2}(\mathbb{R}, \mathbb{R})\right)}^{2} \leqslant 2 T e^{C T}\|h\|_{L^{2}(\mathbb{R}, \mathbb{R})}\|\delta u\|_{C^{0}\left(0, T, H^{1}(\mathbb{R}, \mathbb{R})\right.} .
$$

Combined with the continuity of the map $b \in L^{2} \mapsto u_{b} \in C^{0}\left(0, T, H^{1}(\mathbb{R}, \mathbb{R})\right)$ ensured by Corollary B.1, we can deduce from $(22)$ that $\|\delta u\|_{C^{0}\left(0, T ; L^{2}(\mathbb{R}, \mathbb{R})\right)}^{2}=o\left(\|\delta b\|_{L^{2}(\mathbb{R}, \mathbb{R})}\right)$.

Then, Proposition 4.4 ensures (12) has a unique global solution $v_{b} \in C^{0}\left(0, T ; H^{2}(\mathbb{R}, \mathbb{R})\right)$. Hence, we can correctly compute again the integration-by-parts formula given in $[15$, Lemma 7.3] by considering the Gelfand triple $H^{1}(\mathbb{R}, \mathbb{R}) \subset L^{2}(\mathbb{R}, \mathbb{R}) \subset H^{-1}(\mathbb{R}, \mathbb{R})$ combined with the fact that $\left(\delta u, v_{b}\right) \in\left\{w \in L^{2}\left(0, T ; H^{1}(\mathbb{R}, \mathbb{R})\right): \partial_{t} w \in L^{2}\left(0, T ; H^{-1}(\mathbb{R}, \mathbb{R})\right)\right\}^{2}$. Since $v_{b}(\bullet, T)=\delta u(\bullet, 0)=0$, we have:

$0=\int_{0}^{T}\left\langle\partial_{t}(\delta u) \mid v_{b}\right\rangle_{H^{-1}(\mathbb{R}, \mathbb{R}), H^{1}(\mathbb{R}, \mathbb{R})}(\bullet, t) \mathrm{d} t+\int_{0}^{T}\left\langle\partial_{t} v_{b} \mid \delta u\right\rangle_{H^{-1}(\mathbb{R}, \mathbb{R}), H^{1}(\mathbb{R}, \mathbb{R})}(\bullet, t) \mathrm{d} t$ Proceeding as below (19), one may obtain from the previous relation:

$$
2 \int_{0}^{T} \int_{\mathbb{R}} u_{b} \delta u \mathrm{~d} x \mathrm{~d} t=\int_{0}^{T} \int_{\mathbb{R}} \frac{(\delta u)^{2}}{2} \frac{\partial v_{b}}{\partial x} \mathrm{~d} x \mathrm{~d} t+\int_{0}^{T} \int_{\mathbb{R}} \delta b \frac{\partial v_{b}}{\partial x} \mathrm{~d} x \mathrm{~d} t .
$$

Recalling that $\delta b=h$ and introducing the map (3), we deduce from the last relation:

$R_{F}(h):=F(b+h)-F(b)-\int_{\mathbb{R}} h(x)\left(\int_{0}^{T} \frac{\partial v_{b}}{\partial x}(x, t) \mathrm{d} t\right) \mathrm{d} x=\int_{0}^{T} \int_{\mathbb{R}}(\delta u)^{2}\left(1+\frac{1}{2} \frac{\partial v_{b}}{\partial x}\right) \mathrm{d} x \mathrm{~d} t$.

Consequently, using the fact that $v_{b} \in C^{0}\left(0, T ; H^{2}(\mathbb{R}, \mathbb{R})\right)$, we establish

$$
\left|R_{F}(h)\right| \leqslant T\|\delta u\|_{C^{0}\left(0, T ; L^{2}(\mathbb{R}, \mathbb{R})\right)}^{2}\left(1+\frac{1}{2}\left\|\partial_{x} v_{b}\right\|_{C^{0}\left(0, T ; H^{1}(\mathbb{R}, \mathbb{R})\right)}\right),
$$

and inserting the estimate (22) into the last one above, we get indeed $R_{F}(h)=o\left(\|h\|_{L^{2}(\mathbb{R}, \mathbb{R})}^{2}\right)$. Since $h \in L^{2}(\mathbb{R}, \mathbb{R}) \mapsto \int_{\mathbb{R}} h(x)\left[\int_{[0, T]} \partial_{x} v_{b}(x, t) \mathrm{d} t\right] \mathrm{d} x \in \mathbb{R}$ is a continuous linear form, the uniqueness of the differential ensures that the functional $F_{b}: h \in L^{2}(\mathbb{R}, \mathbb{R}) \mapsto F(b+h) \in \mathbb{R}$ is Fréchet differentiable at the origin i.e. $F$ is Fréchet differentiable at any bottom $b \in L^{2}(\mathbb{R}, \mathbb{R})$ and the shape gradient is well defined by (13), concluding the proof of Proposition 4.5. 


\section{Appendix B Other Useful Properties}

\section{B.1 Hölder Continuity of the Functional}

In $\S 4.3$, given any fixed $K>0$, we have proved the (sequential) continuity of the non-linear $\operatorname{map} N: b \in L^{2}(]-K, K[, \mathbb{R}) \mapsto u_{b} \in C^{0}\left(0, T ; L^{2}(\mathbb{R}, \mathbb{R})\right)$ for the $L^{2}$-weak topology. Here, we first establish $N: L^{2}(\mathbb{R}, \mathbb{R}) \mapsto C^{0}\left(0, T ; H^{1}(\mathbb{R}, \mathbb{R})\right)$ is continuous for the $L^{2}$-strong topology. Then, by restricting $N$ and $F$ to any ball of $L^{2}(\mathbb{R}, \mathbb{R})$, we obtain their Hölder continuity.

Proposition B.1 Let $T>0$ and $b \in L^{2}(\mathbb{R}, \mathbb{R})$. Consider any sequence $\left(b_{n}\right)_{n \in \mathbb{N}}$ of squareintegrable maps strongly converging to $b$ in $L^{2}(\mathbb{R}, \mathbb{R})$. Then, the sequence $\left(u_{b_{n}}\right)_{n \in \mathbb{N}}$ of their associated solutions given in Proposition 4.1 strongly converges to $u_{b}$ in $C^{0}\left(0, T ; H^{1}(\mathbb{R}, \mathbb{R})\right)$, where $u_{b}$ is the unique solution of Proposition 4.1 associated with $b$.

Proof Let $T>0$ and $b \in L^{2}(\mathbb{R}, \mathbb{R})$. From Proposition 4.1, we can consider the unique solution $u_{b} \in C^{0}\left(0, T ; H^{2}(\mathbb{R}, \mathbb{R})\right)$ satisfying $(4)$. First, we treat the smooth case. Let $\left(b_{n}\right)_{n \in \mathbb{N}}$ be a sequence of maps in $H^{\infty}(\mathbb{R}, \mathbb{R})$ that is strongly converging to $b$ for the $L^{2}$-norm. In particular, this sequence is uniformly bounded in $L^{2}(\mathbb{R}, \mathbb{R})$. Applying Proposition A.1, there exists a sequence $\left(u_{b_{n}}\right)_{n \in \mathbb{N}}$ of associated smooth maps satisfying (4) and the a priori estimates, from which we deduce that $\left(u_{b_{n}}\right)_{n \in \mathbb{N}}$ is uniformly bounded in $C^{0}\left(0, T ; H^{2}(\mathbb{R}, \mathbb{R})\right)$ by a constant denoted $C>0$. Then, applying Proposition 4.1 with $b$ and $\mathfrak{b}=b_{n}$ for any $n \in \mathbb{N}$, we obtain that $\left(u_{b_{n}}\right)_{n \in \mathbb{N}}$ strongly converges to $u_{b}$ in $C^{0}\left(0, T ; L^{2}(\mathbb{R}, \mathbb{R})\right)$. We now prove that in fact the convergence occurs in $C^{0}\left(0, T ; H^{1}(\mathbb{R}, \mathbb{R})\right)$. Let $(m, n) \in \mathbb{N} \times \mathbb{N}$. We set $\delta u:=u_{b_{n}}-u_{b_{m}}$ and $\delta b:=b_{n}-b_{m}$ then establish that $\left(u_{b_{k}}\right)_{k \in \mathbb{N}}$ is a uniform Cauchy sequence by relating $\partial_{x}(\delta u)$ to $\delta b$ and $\delta u$. Since both $\left(b_{n}, u_{b_{n}}\right)$ and $\left(b_{m}, u_{b_{m}}\right)$ satisfy $(4)$, we get that $(\delta u, \delta b)$ is a smooth solution to (19) (where we have replaced $u_{b}$ by $u_{b_{m}}$ ). We use the conservative structure of (4) and (19) by writing $\partial_{t}\left(u_{b_{m}}\right)=-\partial_{x} I$ and $\partial_{t}(\delta u)=-\partial_{x} J$, where we set $I:=\partial_{x x} u_{b_{m}}+\frac{1}{2}\left(u_{b_{m}}\right)^{2}+b_{m}$ and $J:=\partial_{x x}(\delta u)+\frac{1}{2}(\delta u)^{2}+\delta b+u_{b_{m}} \delta u$. We have:

$$
\begin{aligned}
& \frac{\mathrm{d}}{\mathrm{d} t} \int_{\mathbb{R}}\left[\frac{(\delta u)^{3}}{6}+u_{b_{m}} \frac{(\delta u)^{2}}{2}-\frac{1}{2}\left(\frac{\partial(\delta u)}{\partial x}\right)^{2}+\delta b \delta u\right]=\underbrace{2 \int_{\mathbb{R}} \partial_{t}(\delta u) J}_{=-\int \partial_{x}\left(J^{2}\right)=0}+\underbrace{\frac{1}{2} \int_{\mathbb{R}} \partial_{t}\left(b_{m}\right)(\delta u)^{2}}_{=-\int I \delta u \partial_{x}(\delta u)} \\
& =-\int_{\mathbb{R}} \delta u \frac{\partial(\delta u)}{\partial x}\left(\frac{\partial^{2} u_{b_{m}}}{\partial x^{2}}+\frac{u_{b_{m}}^{2}}{2}+b_{m}\right) \mathrm{d} x .
\end{aligned}
$$

Proceeding as below (19) (but here the functions are regular), we obtain for any $t \in[0, T]$ :

$$
\begin{aligned}
\| \partial_{x}\left(u_{b_{n}}-u_{b_{m}}\right)(\bullet, t) & \left\|_{L^{2}(\mathbb{R}, \mathbb{R})}^{2} \leqslant \frac{5 C}{3}\right\|\left(u_{b_{n}}-u_{b_{m}}\right)(\bullet, t)\left\|_{L^{2}(\mathbb{R}, \mathbb{R})}^{2}+4 C\right\| b_{n}-b_{m} \|_{L^{2}(\mathbb{R}, \mathbb{R})} \\
& +2 T C\left(C+\frac{C^{2}}{2}+\sup _{k \in \mathbb{N}}\left\|b_{k}\right\|_{L^{2}(\mathbb{R}, \mathbb{R})}\right)\left\|\left(u_{b_{n}}-u_{b_{m}}\right)(\bullet, t)\right\|_{L^{2}(\mathbb{R}, \mathbb{R})} .
\end{aligned}
$$

We deduce from $(23)$ that $t \in[0, T] \mapsto \partial_{x}\left(u_{b_{n}}\right)(\bullet, t) \in L^{2}(\mathbb{R}, \mathbb{R})$ is a uniform Cauchy sequence. It is thus strongly converging to a certain map in $C^{0}\left(0, T ; L^{2}(\mathbb{R}, \mathbb{R})\right)$, which has to be $\partial_{x} u_{b}$ by considering the convergence in the sense of distributions. Finally, we treat the non-regular case by approximations. Let $\varepsilon>0$ and $\left(b_{n}\right)_{n \in \mathbb{N}}$ be any sequence of maps in $L^{2}(\mathbb{R}, \mathbb{R})$ that is strongly converging to $b$. By density, for any $n \in \mathbb{N}$, there exists a sequence $\left(b_{n}^{k}\right)_{k \in \mathbb{N}}$ of smooth maps with compact support that is strongly converging to $b_{n}$ in $L^{2}(\mathbb{R}, \mathbb{R})$. From the foregoing, we deduce that there exists $k_{n} \in \mathbb{N}$ such that we have $\left\|u_{b_{n}^{k_{n}}}-u_{b_{n}}\right\|_{C^{0}\left(0, T ; H^{1}(\mathbb{R}, \mathbb{R})\right)}<\varepsilon$. Moreover, one can check that $\left(b_{n}^{k_{n}}\right)_{n \in \mathbb{N}}$ strongly converges to $b$ in $L^{2}(\mathbb{R}, \mathbb{R})$. Again, from the foregoing, there exists $N \in \mathbb{N}$ such that for any integer $n \geqslant N$, we have $\left\|u_{b_{n}^{k_{n}}}-u_{b}\right\|_{C^{0}\left(0, T ; H^{1}(\mathbb{R}, \mathbb{R})\right)}<\varepsilon$. We deduce for any $n \geqslant N$ :

$\left\|u_{b_{n}}-u_{b}\right\|_{C^{0}\left(0, T ; H^{1}(\mathbb{R}, \mathbb{R})\right)} \leqslant\left\|u_{b_{n}}-u_{b_{n}^{k_{n}}}\right\|_{C^{0}\left(0, T ; H^{1}(\mathbb{R}, \mathbb{R})\right)}+\left\|u_{b_{n}^{k_{n}}}-u_{b}\right\|_{C^{0}\left(0, T ; H^{1}(\mathbb{R}, \mathbb{R})\right)}<2 \varepsilon$.

To conclude the proof of Proposition B.1, $\left(u_{b_{n}}\right)$ converges to $u_{b}$ in $C^{0}\left(0, T ; H^{1}(\mathbb{R}, \mathbb{R})\right)$. 
Corollary B.1 Let $M>0, T>0$ and set $B_{M}:=\left\{b \in L^{2}(\mathbb{R}, \mathbb{R}):\|b\|_{L^{2}(\mathbb{R}, \mathbb{R})} \leqslant M\right\}$. Then, there exists $C(T, M)>0$ depending only on $T$ and $M$ such that for any $(b, \mathfrak{b}) \in B_{M} \times B_{M}$ :

$$
\left\{\begin{array}{l}
\max \left(\left\|u_{b}-u_{\mathfrak{b}}\right\|_{C^{0}\left(0, T ; L^{2}(\mathbb{R}, \mathbb{R})\right)},|F(b)-F(\mathfrak{b})|\right) \leqslant C(T, M) \sqrt{\|b-\mathfrak{b}\|_{L^{2}(\mathbb{R}, \mathbb{R})}} \\
\left\|u_{b}-u_{\mathfrak{b}}\right\|_{C^{0}\left(0, T ; H^{1}(\mathbb{R}, \mathbb{R})\right)} \leqslant C(T, M) \sqrt[4]{\|b-\mathfrak{b}\|_{L^{2}(\mathbb{R}, \mathbb{R})}} \cdot
\end{array}\right.
$$

In particular, the energy functional $F: \mathcal{B} \rightarrow \mathbb{R}$ given in (3) is $\frac{1}{2}$-Hölder continuous.

Proof Let $M>0, T>0$, and set $B_{M}:=\left\{b \in L^{2}(\mathbb{R}, \mathbb{R}):\|b\|_{L^{2}(\mathbb{R}, \mathbb{R})} \leqslant M\right\}$. First, we combine the a priori estimate of Proposition 4.2 with the fact that $(b, \mathfrak{b}) \in B_{M} \times B_{M}$. We deduce that the constant $C>0$ appearing in the quantitative estimate of Proposition 4.1 can be bounded by one that only depends on $T$ and $M$. Hence, the non-linear map $N: b \in B_{M} \mapsto u_{b} \in C^{0}\left(0, T ; L^{2}(\mathbb{R}, \mathbb{R})\right)$ is $\frac{1}{2}$-Hölder continuous. Then, the continuity of the embedding $C^{0}\left(0, T ; L^{2}(\mathbb{R}, \mathbb{R})\right) \subset L^{2}\left(0, T ; L^{2}(\mathbb{R}, \mathbb{R})\right)$ applied to $b$ and $b_{n^{\prime}}=\mathfrak{b}$ also yields to the same result for the map $F: B_{M} \mapsto \mathbb{R}$. Finally, there exists two sequences $\left(b_{n}\right)_{n \in \mathbb{N}}$ and $\left(\mathfrak{b}_{n}\right)_{n \in \mathbb{N}}$ of smooth maps with compact support respectively converging to $b$ and $\mathfrak{b}$ strongly in $L^{2}(\mathbb{R}, \mathbb{R})$. Proposition B.1 ensures that the associated smooth maps $\left(u_{b_{n}}\right)_{n \in \mathbb{N}}$ and $\left(u_{\mathfrak{b}_{n}}\right)_{n \in \mathbb{N}}$ respectively converges to $u_{b}$ and $u_{\mathfrak{b}}$ in $C^{0}\left(0, T ; H^{1}(\mathbb{R}, \mathbb{R})\right)$. We can now proceed as in the proof of Proposition B.1 so (23) holds with $b_{n}$ and $b_{m}=\mathfrak{b}_{n}$. By letting $n \rightarrow+\infty$ in this inequality, we deduce from the foregoing that $N: b \in B_{M} \mapsto u_{b} \in C^{0}\left(0, T ; H^{1}(\mathbb{R}, \mathbb{R})\right)$ is $\frac{1}{4}$-Hölder continuous, concluding the proof of Corollary B.1.

\section{B.2 Stability Analysis of the Numerical Scheme}

Proposition B.2 The discretization (15) takes the form $L_{\Delta x, \Delta t} u=0$ and approximates equation (6) written as $\partial_{t} u+L u=0$. If we assume $\Delta t=O(\Delta x)$, then (15) is consistent and first-order accurate: $\forall u \in C^{4}\left(\mathbb{R} \times\left[0,+\infty[, \mathbb{R}), \partial_{t} u+L u=0 \Rightarrow \partial_{t} u+L u=L_{\Delta x, \Delta t} u+O(\Delta x)\right.\right.$.

Proof We introduce the shift operators $s_{x}^{ \pm}[(\bullet)(x, t)]:=(\bullet)(x \pm \Delta x, t)$ in order to define $\delta_{x}^{1}:=\frac{1}{2 \Delta x}\left(s_{x}^{+}-s_{x}^{-}\right), \delta_{x}^{2}:=\frac{1}{\Delta x^{2}}\left(s_{x}^{+}-2+s_{x}^{-}\right)$and $\delta_{x}^{3}:=\delta_{x}^{1} \delta_{x}^{2}$. Let $u \in C^{4}(\mathbb{R} \times[0,+\infty[, \mathbb{R})$ be such that $\partial_{t} u+L u=0$. We get $s_{x}^{ \pm} u=u \pm \Delta x \partial_{x} u+\frac{\Delta x^{2}}{2} \partial_{x x} u \pm \frac{\Delta x^{3}}{6} \partial_{x x x} u+O\left(\Delta x^{4}\right)$ from a Taylor expansion. We deduce $\partial_{x} u=\delta_{x}^{1} u+O\left(\Delta x^{2}\right)$ and $\partial_{x x} u=\delta_{x}^{2} u+O\left(\Delta x^{2}\right)$. These estimations are then combined to obtain $\partial_{x x x} u=\delta_{x}^{3} u+O(\Delta x)$. Therefore, we have an approximation of the linear terms of $L$ :

$$
L u=\frac{3 c_{0}}{2 h_{0}} u \partial_{x} u+\frac{c_{0} h_{0}^{2}}{6} \delta_{x}^{3} u+\frac{c_{0}}{2} \delta_{x}^{1} b+O(\Delta x) .
$$

Introducing the time operators $s_{t}^{+}[(\bullet)(x, t)]:=(\bullet)(x, t+\Delta t)$ and $\delta_{t}^{+}:=\frac{1}{\Delta t}\left(s_{t}^{+}-1\right)$, similar arguments yields $\partial_{t} u=\delta_{t}^{+} u+O(\Delta t)$ and $\partial_{t} u=s_{t}^{+} \partial_{t} u+O(\Delta t)=-s_{t}^{+} L u+O(\Delta t)$. We get: $\partial_{t} u+L u=\delta_{t}^{+} u+L u+O(\Delta t)=\delta_{t}^{+} u+\frac{1}{2}\left(L u-\partial_{t} u\right)+O(\Delta t)=\delta_{t}^{+} u+\frac{1}{2}\left(1+s_{t}^{+}\right) L u+O(\Delta t)$. Then, we assume $\Delta t=O(\Delta x)$ and from $\frac{1}{2}\left(s_{t}^{+}+1\right) u^{2}=u s_{t}^{+} u+O\left(\Delta t^{2}\right)$, we can treat the non-linear term of $L u$ : $\left(1+s_{t}^{+}\right)\left(u \partial_{x} u\right)=\delta_{x}^{1}\left[u s_{t}^{+} u+O\left(\Delta t^{2}\right)\right]+O\left(\Delta x^{2}\right)=\delta_{x}^{1}\left(u s_{t}^{+} u\right)+O(\Delta x)$. Hence, we deduce the expected estimation $\partial_{t} u+L u=L_{\Delta x, \Delta t} u+O(\Delta x)$ with:

$$
L_{\Delta x, \Delta t} u:=\delta_{t}^{+} u+\frac{3 c_{0}}{4 h_{0}} \delta_{x}^{1}\left(u s_{t}^{+} u\right)+\frac{c_{0} h_{0}^{2}}{12}\left(1+s_{t}^{+}\right)\left(\delta_{x}^{3} u\right)+\frac{c_{0}}{2} \delta_{x}^{1} b .
$$

Proposition B.3 Consider the discretization (15) of equation (6) with forcing term $b=0$. Let $\beta=\frac{3 c_{0}}{2 h_{0}}\|\zeta\|_{C^{0}([-L, L] \times[0, T], \mathbb{R})}, \mu=\frac{c_{0}}{6} h_{0}^{2}$ and $s=\frac{\Delta t}{\Delta x}$. Then, Von Neumann's stability analysis provides an amplification factor $g:[-\pi, \pi] \rightarrow \mathbb{C}$ of the following form:

$$
g(\xi):=\frac{1-i A(\xi)}{1+i A(\xi)} \quad \text { where } \quad A(\xi):=s(\sin \xi)\left[\frac{\beta}{2}+\frac{\mu}{\Delta x^{2}}(\cos \xi-1)\right] .
$$


In particular, $|g|=1$, ensuring the non-dissipative feature of the method: the scheme (15) is unconditionally stable. Moreover, the numerical dispersion $\Psi=\arg (g)=-\arctan \left(\frac{2 A}{1-A^{2}}\right)$ is compared to the analytical one whose expression is given by $\Psi_{r e f}(\xi):=-s \beta \xi+\frac{s \mu}{\Delta x^{2}} \xi^{3}$. We obtain $\Psi(\xi)=\Psi_{\text {ref }}(\xi)+E_{\Psi}(\xi)+O\left(\xi^{7}\right)$ where:

$$
E_{\Psi}(\xi)=\frac{s \beta}{6}\left(1+\frac{s^{2} \beta^{2}}{2}\right) \xi^{3}-\left[\frac{s^{5} \beta^{5}}{80}+\frac{s^{3} \beta^{3}}{24}+\frac{s \beta}{120}+\frac{s^{3} \beta^{2} \mu}{4 \Delta x^{2}}+\frac{s \mu}{4 \Delta x^{2}}\right] \xi^{5} .
$$

Proof We refer to [23, (38)-(51)] for details on the proof of this result. We stress, however, a disagreement between our expression of $E_{\Psi}$ and the one provided in [23, (50)]. This seems to result from a mistake made in $[23,(48)]$, when expanding $\Psi$ by using Taylor series. More precisely, it is wrongly stated that $\arctan \left(\frac{-2 A}{1-A^{2}}\right)=-2 A\left[1-\frac{1}{3} A^{2}-3 A^{4}\right]+O\left(A^{7}\right)$, as the correct expression is given by $\arctan \left(\frac{-2 A}{1-A^{2}}\right)=-2 A\left[1-\frac{1}{3} A^{2}+\frac{1}{5} A^{4}\right]+O\left(A^{7}\right)$.

\section{B.3 The Necessity of a $L^{2}$-Constraint}

Lemma B.1 Let $b(x)$ be a forcing function with enough regularity, as given in Lemma 3.1, and $u(x, t)$ be the unique smooth solution of the initial value problem (4). For any $\theta \in \mathbb{R}$, define the maps $u_{\theta}:(x, t) \mapsto \theta^{2} u\left(\theta x, \theta^{3} t\right)$ and $b_{\theta}: x \mapsto \theta^{4} b(\theta x)$. Then, $u_{\theta}$ is precisely the solution of (4) with forcing function $b_{\theta}$.

Proposition B.4 Let $K>0$ and $T>0$. Then, the problem (11) has no global maximizer.

Proof Assume, by contradiction, that there exists a maximizer $b$ to (11). Then, from Lemma 3.1 , we can consider its associated smooth solution $u_{b}$. Introducing the bottoms $\left(b_{\theta}\right)_{\theta>1}$ of Lemma B.1, one can check they are admissible for problem (11). Moreover, we deduce from Lemma B.1 that $F\left(b_{\theta}\right)=\int_{0}^{\theta^{3} T} \int_{\mathbb{R}} u_{b}^{2}(x, t) \mathrm{d} x \mathrm{~d} t$. Using the optimality of $b$, we obtain $F\left(b_{\theta}\right)=F(b)$ for any $\theta>1$ so $u_{b}=0$ on $\left[T, \theta^{3} T\right]$. From (6), we get $\partial_{x} b=0$ thus $u_{b}=0$ also on $[0, T]$. Thus $F(b)=0$, which contradicts the optimality of $b$.

\section{References}

1. Wu, T.Y., Wu, D.M.: Three-dimensional nonlinear long waves due to moving surface pressure. In: Proceedings of the 14th Symposium on Naval Hydrodynamics, 103-125. National Academy Press (1982)

2. Instant Surfing. Available at http://www.wavegarden.com (2012)

3. Wu, T.Y.: Generation of upstream advancing solitons by moving disturbances. Journal of Fluid Mechanics 184, 75-99 (1987)

4. Cao, Y., Beck, R.F., Schultz, W.W.: Numerical computations of two-dimensional solitary waves generated by moving disturbances. International Journal for Numerical Methods in Fluids 17, 905-920 (1993)

5. Lee, S.J., Yates, G.T., Wu, T.Y.: Experiments and analyses of upstream-advancing solitary waves generated by moving disturbances. Journal of Fluid Mechanics 199, 569-593 (1989)

6. Zhang, D., Chwang, A.T.: Numerical study of nonlinear shallow water waves produced by a submerged moving disturbance in viscous flow. Physics of Fluids 8, 147-155 (1996)

7. Bona, J.L., Zhang, B.Y.: The initial-value problem for the forced Korteweg-de Vries equation. Proceedings of the Royal Society of Edinburgh Series A 126, 571-598 (1996)

8. Colliander, J., Keel, M., Staffilani, G., Takaoka, H., Tao, T.: Sharp global well-posedness for $\mathrm{KdV}$ and modified $\mathrm{KdV}$ on $\mathbb{R}$ and $\mathbb{T}$. Journal of the American Mathematical Society $16,705-749$ (2003)

9. Tsugawa, K.: Global well-posedness for the KdV equations on the real line with low regularity forcing terms. Communications in Contemporary Mathematics 8, 681-713 (2006) 
10. Kenig, C.E., Ponce, G., Vega, L.: A bilinear estimate with applications to the KdV equation. Journal of the American Mathematical Society 9, 573-603 (1996)

11. Rosier, L., Zhang, B.Y.: Control and stabilization of the Korteweg-de Vries equation: recent progresses. Journal of Systems Science and Complexity 22, 647-682 (2009)

12. Nersisyan, H., Dutykh, D., Zuazua, E.: Generation of $2 \mathrm{D}$ water waves by moving disturbances. IMA Journal of Applied Mathematics 80, 1235-1253 (2015)

13. Bona, J.L., Chen, M., Saut, J.-C.: Boussinesq equations and other systems for smallamplitude long waves in nonlinear dispersive media II. Nonlinearity 17, 925-952 (2004)

14. Simon, J.: Compact sets in the space $L^{p}(0, T ; B)$. Annali di Matematica Pura ed Applicata. 146, 65-96 (1987)

15. Roubíček, T.: Nonlinear partial differential equations with applications. In: International Series of Numerical Mathematics, volume 153. Birkhäuser (2005)

16. Zabusky, N.J., Kruskal, M.D.: Interaction of solitons in a collisionless plasma and the recurrence of initial states. Physical Review Letters 15, 240-243 (1965)

17. Fornberg, B., Whitham, G.B.: A numerical and theoretical study of certain nonlinear wave phenomena. Philosophical Transactions of the Royal Society of London, Series A, Mathematical and Physical Sciences 289, 373-404 (1978)

18. Trefethen, L.N.: Spectral method in Matlab, chapter 10. Society for Industrial and Applied Mathematics (2000)

19. Drazin, P.G., Johnson, R.S.: Solitons: an introduction. In: Cambridge Texts in Applied Mathematics, 2nd edition. Cambridge University Press (1989)

20. Trélat, E., Zuazua, E.: The turnpike property in finite-dimensional nonlinear optimal control. Journal of Differential Equations 258 (2015)

21. Furihata, D.: Finite difference schemes for $\frac{\partial u}{\partial t}=\left(\frac{\partial}{\partial x}\right)^{\alpha} \frac{\delta g}{\delta u}$ that inherit energy conservation or dissipation property. Journal of Computational Physics 156, 181-205 (1999)

22. Camassa, R., Wu, T.Y.: Stability of forced steady solitary waves. Philosophical Transactions of the Royal Society of London A: Mathematical, Physical, and Engineering Sciences 337, 429-466 (1991)

23. Djidjeli, K., Pride, W.G., Twizell, E.H., Wang Y.: Numerical methods for the solution of the third and fifth-order dispersive Korteweg-de Vries equations. Journal of Computational and Applied Mathematics 58, 307-336 (1995) 